\title{
Article
}

\section{Cointegration, Root Functions and Minimal Bases}

\author{
Massimo Franchi ${ }^{1, *(D)}$ and Paolo Paruolo ${ }^{2}$ (D) \\ 1 Department of Statistical Sciences, Sapienza University of Rome, P.le A. Moro 5, 00185 Rome, Italy \\ 2 European Commission Joint Research Centre, Via E. Fermi 2749, 21027 Ispra, Italy; \\ paolo.paruolo@ec.europa.eu \\ * Correspondence: massimo.franchi@uniroma1.it
}

check for

updates

Citation: Franchi, Massimo, and Paolo Paruolo. 2021. Cointegration, Root Functions and Minimal Bases. Econometrics 9: 31. https://doi.org/ 10.3390/econometrics 9030031

Academic Editor: Rocco Mosconi

Received: 9 December 2019

Accepted: 13 August 2021

Published: 17 August 2021

Publisher's Note: MDPI stays neutral with regard to jurisdictional claims in published maps and institutional affiliations.

Copyright: (c) 2021 by the authors. Licensee MDPI, Basel, Switzerland. This article is an open access article distributed under the terms and conditions of the Creative Commons Attribution (CC BY) license (https:/ / creativecommons.org/licenses/by/ $4.0 /)$.

\begin{abstract}
This paper discusses the notion of cointegrating space for linear processes integrated of any order. It first shows that the notions of (polynomial) cointegrating vectors and of root functions coincide. Second, it discusses how the cointegrating space can be defined (i) as a vector space of polynomial vectors over complex scalars, (ii) as a free module of polynomial vectors over scalar polynomials, or finally (iii) as a vector space of rational vectors over rational scalars. Third, it shows that a canonical set of root functions can be used as a basis of the various notions of cointegrating space. Fourth, it reviews results on how to reduce polynomial bases to minimal order-i.e., minimal bases. The application of these results to Vector AutoRegressive processes integrated of order 2 is found to imply the separation of polynomial cointegrating vectors from non-polynomial ones.
\end{abstract}

Keywords: VAR; cointegration; I(d); vector spaces

\section{Introduction}

In their seminal paper, Engle and Granger (1987) introduced the notion of cointegration and of cointegrating (CI) rank for processes integrated of order 1, or I(1). They did this in the following way: ${ }^{1}$

DEFINITION: The components of the vector $x_{t}$, are said to be co-integrated of order $d, b$, denoted $x_{t} \sim C I(d, b)$, if (i) all components of $x_{t}$, are $I(d)$; (ii) there exists a vector $\beta(\neq 0)$ so that $z_{t}=\beta^{\prime} x_{t} \sim I(d-b), b>0$. The vector $\beta$ is called the co-integrating vector.

[...] If $x_{t}$ has $p$ components, then there may be more than one co-integrating vector $\beta$. It is clearly possible for several equilibrium relations to govern the joint behavior of the variables. In what follows, it will be assumed that there are exactly $r$ linearly independent co-integrating vectors, with $r \leq p-1$, which are gathered together into the $p \times r$ array $\beta$. By construction the rank of $\beta$ will be $r$ which will be called the "co-integrating rank" of $x_{t}$.

Engle and Granger (1987) did not define explicitly the notion of cointegrating space, but just the cointegrating rank, which corresponds to its dimension; explicit mention of the cointegrating space was first made in Johansen (1988).

The Granger representation theorem in Engle and Granger (1987) showed that the cointegration matrix $\beta$ needs to be orthogonal to the Moving Average (MA) impact matrix of $\Delta x_{t}$. More precisely, for $\Delta x_{t}=C(L) \varepsilon_{t}$, the MA impact matrix $C(1)$ has rank equal to $p-r$ and representation $C(1)=\beta_{\perp} a^{\prime}$, where $\beta_{\perp}$ is a basis of the orthogonal complement of the space spanned by the columns of $\beta$ and $a$ is full column rank.

Johansen $(1991,1992)$ stated the appropriate conditions under which the Granger representation theorem holds for I(1) and I(2) Vector AutoRegressive processes (VAR) $A(L) x_{t}=\varepsilon_{t}$, where the AR impact matrix $A(1)$ has rank equal to $r<p$ and rank factorization $A(1)=-\alpha \beta^{\prime}$, with $\alpha$ and $\beta$ of full column rank. He defined the cointegrating space as the vector space generated by the column vectors $\beta_{j}$ in $\beta$ over the field of real numbers $\mathbb{R}$. 
Johansen (1991) noted that $\mathcal{B}=\operatorname{row}_{\mathbb{R}}\left(\beta^{\prime}\right)$ is uniquely defined ${ }^{2}$ by the rank factorization $A(1)=-\alpha \beta^{\prime}$, but the choice of basis $\beta^{\prime}$ is arbitrary, i.e., $\beta^{\prime}$ is not identified. Hypotheses that do not constrain $\mathcal{B}$ are hence untestable. He proposed likelihood ratio tests on $\mathcal{B}$ and described asymptotic properties of a just-identified version of $\beta^{\prime}$. Later Johansen (1995) discussed the choice of basis $\beta^{\prime}$ as an econometric identification problem of a system of simultaneous equations (SSE) of cointegrating relations describing the long-run equilibria in the process. He discussed identification using linear restrictions, along the lines of the classical identification problem of SSE studied in econometrics since the early days of the Cowles Commission.

The observation in Johansen (1988) that the cointegrating vectors formed a vector space $\mathcal{B}$ was an important breakthrough. For instance, it addressed the question: 'How many cointegrating vectors should one estimate in a given system of dimension $p$ ?'. A proper answer is in fact: A set of $r$ linearly independent vectors, spanning the cointegrating space $\mathcal{B}$, i.e., a basis of $\mathcal{B}$.

Similarly, when assuming that a set of $p$ interest rates is described by an I(1) process, the notion of cointegrating space $\mathcal{B}$ enables one to discuss questions like 'How should one test that all interest rates spreads are stationary?'. In fact, if all $\left(\begin{array}{c}p \\ 2\end{array}\right)=p(p-1) / 2$ interest rates differentials were stationary, then one should have cointegrating rank $r=p-1$, which gives a first testable hypothesis on the cointegrating rank. Moreover there is no need to test all possible interest rates differentials to be stationary, but, if the cointegrating rank has been found to be $p-1$, one can test that the cointegrating space is spanned by any set of linearly independent $r$ contrasts between pairs of interest rates. If the cointegrating rank is found to be $0<r<p-1$, one may still want to test the restriction that the cointegrating space $\mathcal{B}$ is a subspace of the linear space spanned by all contrasts.

These questions, and many more, found clear answers thanks to the introduction of the notion of cointegrating space. The recognition that the set of cointegrating vectors forms a vector space was then instrumental to represent any cointegrating vector as a linear combination of the ones in a basis of the vector space.

The notion of cointegrating space, together with the complementary notion of attractor space, has been recently discussed in the context of functional time series for infinite dimensional Hilbert space valued AR processes with unit roots, see Beare et al. (2017), Beare and Seo (2020), Franchi and Paruolo (2020), and for infinite dimensional Banach space valued AR processes with unit roots, see Seo (2019).

For systems with variables integrated of order $d, I(d)$, with $d=2,3, \ldots$ Granger and Lee (1989) and Engle and Yoo (1991) introduced the related notions of multicointegration and polynomial cointegration; see also Engsted and Johansen (2000). However, no proper discussion of cointegrating spaces or of their corresponding bases has been proposed in the literature for higher order systems.

The present paper closes this gap, making use of classical concepts in local spectral theory, see Gohberg et al. (1993). A central role is played by canonical system of root functions, which have already been exploited in Franchi and Paruolo $(2011,2016)$ to characterize the inversion of a matrix function, and used in Franchi and Paruolo (2019) to derive the generalization of the Granger-Johansen representation theorem for $\mathrm{I}(d)$ processes.

In order to simplify exposition, this paper focuses on unit roots at a single point $z_{\omega}$, indexed by frequency $\omega$. When $\omega \notin\{0, \pi\}$, the resulting matrices are complex-valued, and the symbol $F$ is taken to indicate $\mathbb{C}$. For $\omega \in\{0, \pi\}, F$ is taken instead to indicate $\mathbb{R}$. Unit roots at distinct seasonal frequencies different from 0 have been considered e.g., in Hylleberg et al. (1990), Gregoir (1999), Johansen and Schaumburg (1998), Bauer and Wagner (2012). Several of these papers paired frequencies $\pm \omega$ when $\omega \notin\{0, \pi\}$ to obtain real coefficient matrices for Equilibrium Correction (EC) representations; in order to keep exposition as simple as possible, this is not attempted in the present paper.

To the best of the authors' knowledge, local spectral theory tools are employed here for the first time to discuss the definition of cointegrating space for $I(d)$ processes, $d>1$, and related bases. It is observed that several candidate cointegrating spaces exists, corre- 
sponding to different choices of the set of vectors and scalars. The sets of vectors are chosen here to be either the set of polynomial vectors or the one of rational vectors, while the set of scalars are taken to be (i) the field $F=\mathbb{R}, \mathbb{C}$, (ii) the ring of polynomials with coefficients in $F$ (denoted $F[z]$ ) or (iii) the field of rational function with coefficients in $F$ (denoted $F(z)$ ). The resulting spaces are either vector spaces, in cases (i) and (iii), or a free module in case (ii). The relationship among their bases is discussed following Forney (1975), whose results are used to derive a polynomial basis of minimal degree-i.e., a minimal basis.

The focus of this paper is on the parsimonious representation of the set of cointegrating vectors. As noted by a referee, the present results may find application also in the parametrization and estimation of $I(d)$ EC systems. This, however, is beyond the scope of the present paper.

The rest of the paper is organised as follows. Section 2 provides the motivation for the paper. Section 3 reports definitions of integration and cointegration in $I(d)$ systems, where the cointegrating vectors $\zeta(z)^{\prime}=\sum_{j=0}^{\infty}\left(z-z_{\omega}\right)^{j} \zeta_{j}^{\prime}$ are allowed to be vector functions; here $\left(z-z_{\omega}\right)$ and its powers are associated with the difference operator and its powers. Section 4 defines root functions and canonical systems of root functions and Section 5 discusses possible definitions of the cointegration space. Section 6 discusses how to derive bases for the various notions of cointegrating space from VAR coefficients. Section 7 discusses minimal bases using results in Forney (1975) and Section 8 applies these results in order to obtain a minimal basis in the I(2) VAR case. Section 9 concludes; Appendix A reports background results.

\section{Motivation}

This section motivates the study of the represention of cointegrating vectors in terms of bases of suitable spaces, for systems integrated of order two, which are more formally introduced in Section 3 below. Let $x_{t}$ be a $p \times 1$ vector process, and let $\Delta=1-L$ and $L$ be the (0-frequency) difference and the lag operators. Assume that $x_{t}$ is integrated of order 2, $\mathrm{I}(2)$, with $\Delta^{j} x_{t}$ nonstationary for $j<2$ and stationary for $j \geq 2$.

Mosconi and Paruolo (2017) consider the identification problem for the following cointegrating SSE with $I(2)$ variables

$$
\operatorname{ecm}_{t}=\xi(\Delta)^{\prime} x_{t}, \quad \text { with } \quad \xi(\Delta)^{\prime}:=\left(\begin{array}{c}
\beta^{\prime}+v^{\prime} \Delta \\
\gamma^{\prime} \Delta \\
\beta^{\prime} \Delta
\end{array}\right) \begin{aligned}
& \} \\
& \} \\
& r_{0} \\
& r_{1} \\
& r_{0}
\end{aligned}
$$

The first set of $r_{0}$ polynomial vectors has coefficient $\beta^{\prime}$ of order 0 (i.e., that multiplies $\Delta^{0}$ ) and coefficient $v^{\prime}$ of order 1 (i.e., that multiplies $\Delta^{1}$ ). The last $r_{0}+r_{1}$ polynomial vectors have 0 coefficients of order 0 and $\gamma^{\prime}$ and $\beta^{\prime}$ coefficients of order 1 . They discussed identification of the SSE with respect to transformations corresponding to pre-multiplication of $\xi(\Delta)^{\prime}$ (or $\mathrm{ecm}_{t}$ ) by a block triangular, nonsingular matrix of the form

$$
Q=\left(\begin{array}{ccc}
Q_{00} & Q_{0 \gamma} & Q_{0 \beta} \\
0 & Q_{\gamma \gamma} & Q_{\gamma \beta} \\
0 & 0 & Q_{00}
\end{array}\right)
$$

where $Q_{a b}$ are blocks of real coefficients, $a, b \in\{0, \gamma, \beta\}$, with $Q_{00}$ and $Q_{\gamma \gamma}$ nonsingular square matrices.

They show that $Q \xi(\Delta)^{\prime}=\xi^{\circ \prime}(\Delta)$ has the same structure as $\xi(\Delta)^{\prime}$ in terms of the null coefficient of order 0 in the last $r_{1}+r_{0}$ equations, as well as the same $\beta$ block as the coefficient of order 0 in the first $r_{0}$ and as the coefficient of order 1 in the last $r_{0}$ rows. More precisely,

- $\quad \beta^{\prime}$ is replaced by $\beta^{\circ \prime}=Q_{00} \beta^{\prime}$, a set of $r_{0}$ linear combinations of $\beta^{\prime}$,

- $\gamma^{\prime} \Delta$ is replaced by a set of $r_{1}$ linear combinations of $\gamma^{\prime} \Delta$ and $\beta^{\prime} \Delta$,

- $v^{\prime} \Delta$ is replaced by a set of $r_{0}$ linear combinations of $v^{\prime} \Delta, \gamma^{\prime} \Delta$ and $\beta^{\prime} \Delta$. 
Remark 1 (F-linear combinations). Note that the $Q$ linear combinations have scalars taken from $F=\mathbb{R}$, and that any $C I$ vectors can be obtained as linear combinations with coefficients in $F$ of the rows in $\xi(\Delta)^{\prime}$, called in the following 'F-linear combinations'.

The main motivation to study the notion of cointegration space for $I(d)$ processes with $d \geq 2$ comes from the following observation.

Remark $2(F[\Delta]$-linear combinations). The set of $C I$ vectors obtained as F-linear combinations of the rows in $\xi(\Delta)^{\prime}$ can be also obtained by considering the alternative set of cointegrating vectors

$$
\left.\zeta(\Delta)^{\prime}:=\left(\begin{array}{c}
\beta^{\prime}+v^{\prime} \Delta \\
\gamma^{\prime}
\end{array}\right)\right\} \begin{aligned}
& \gamma r_{0} \\
& \} r_{1}
\end{aligned}
$$

and choosing linear combinations with scalar in the set of polynomials $F[\Delta]$, where $a(z) \in F[z]$ has the form $a(z)=\sum_{j=0}^{n} a_{j} z^{j}$ for some finite $n$.

To show that the set of $F[\Delta]$-linear combinations of $\zeta(\Delta)^{\prime}$ is the same as the set of F-linear combinations of $\xi(\Delta)^{\prime}$, it is sufficient to show that the rows of $\xi(\Delta)^{\prime}$ can be obtained as $F[\Delta]$-linear combinations of the rows in $\zeta(\Delta)^{\prime}$, possibly up to terms of the type $c^{\prime} \Delta^{2}$ which generate stationary processes by definition.

Note first that $\beta^{\prime}+v^{\prime} \Delta$ is common to $\xi(\Delta)^{\prime}$ and $\zeta(\Delta)^{\prime}$. In order to obtain $\gamma^{\prime} \Delta$ in $\xi(\Delta)^{\prime}$ from $\zeta(\Delta)^{\prime}$ one needs to select the scalar $\Delta$ from $F[\Delta]$ and multiply it by $\gamma^{\prime}$. Similarly, in order to obtain $\beta^{\prime} \Delta$ in $\xi(\Delta)^{\prime}$ one only needs to select the scalar $\Delta \in F[\Delta]$ and multiply it by $\beta^{\prime}+v^{\prime} \Delta$ to obtain $\beta^{\prime} \Delta+v^{\prime} \Delta^{2}$. Because $\Delta^{2} x_{t}$ is stationary by the assumption that $x_{t}$ is I(2), the term $v^{\prime} \Delta^{2}$ can be discarded, and this completes the argument.

The take-away from Remark 2 is that, if one allows the set of multiplicative scalars to contain polynomials, i.e., if one moves from $F$-linear combinations to $F[\Delta]$-linear combinations, then one can reduce the number of rows needed to generate the set of CI vectors: $\xi(\Delta)^{\prime}$ in fact has $2 r_{0}+r_{1}$ rows, while the number of rows in $\zeta(\Delta)^{\prime}$ is $r_{0}+r_{1}$.

The previous discussion shows that the two sets, $F$ and $F[\Delta]$, could be used as possible set of scalars in taking linear combinations. The first one, $F$, is a field (i.e., a division ring), the second one, $F[\Delta]$, is a ring but not a field because it lacks the multiplicative inverse.

Given that vector spaces require the set of scalars to be a field, one may also consider another possible set of scalars, namely $F(\Delta)$, the set of rational functions of the type $a(\Delta)=c(\Delta) / d(\Delta)$ with $c(\Delta), d(\Delta) \in F[\Delta]$, and $d(\Delta)$ not identically equal to 0 , indicated as $d(\Delta) \not \equiv 0$. This leads to consider three possible choices for the set of scalars: (i) The field $F$, (ii) the ring $F[\Delta]$ and (iii) the field $F(\Delta)$. The rest of the paper discusses relative merits of using any of them.

The above discussion focused on unit roots at $z=1$, which are associated to the long run behavior of the process. When data are observed every month or quarter, seasonal unit roots, seasonal cointegration and seasonal error correction have been shown to be useful notions, see Hylleberg et al. (1990). For instance, in the case of quarterly series, the relevant seasonal unit roots are at $z=-1$ and at $z \pm i$ where $i$ is the imaginary unit. These roots are represented as $z_{\omega}=\exp (i \omega)$ with $0 \leq \omega<2 \pi$, where $z_{\omega}=1, i,-1,-i$ correspond to $\omega=0, \frac{1}{2} \pi, \pi, \frac{3}{2} \pi$.

Johansen and Schaumburg (1998) showed that the conditions under which a VAR process allows for seasonal integration (and cointegration) of order 1 are of the same type as for roots at $z=1$, except that expansions of the VAR polynomial are performed around each $z_{\omega}$, see their Theorem 3. They also provided the corresponding EC form in their Corollary 2; see also Bauer and Wagner (2012) and the discussion in Remark 9 below.

In general, the conditions for integration of any order $d$ at a point $z_{\omega}$ on the unit circle can be shown to be of the same type. This paper hence considers the generic case of a linear process with a generic root on the unit circle $z_{\omega}=\exp (i \omega)$, and discusses the notions of cointegration, root functions and minimal bases in this general context. This allows to show that the present results hold for generic frequency $\omega, 0 \leq \omega<2 \pi$. 
Incidentally, the results presented below in Section 6 state the generalization of the Granger and the Johansen Representation Theorems presented in Franchi and Paruolo (2019) for a generic unit root $z_{\omega}=\exp (i \omega)$ at any frequency $\omega$.

\section{Setup and Definitions}

This section introduces notation and basic definitions of integrated and cointegrated processes.

\subsection{Linear Processes}

Assume that $\left\{\varepsilon_{t}, t \in \mathbb{Z}\right\}$ is a $p \times 1$ i.i.d. sequence, called a noise process, ${ }^{3}$ with $\mathrm{E}\left(\varepsilon_{t}\right)=0$ and $\mathrm{E}\left(\varepsilon_{t} \varepsilon_{s}^{\prime}\right)=\Omega 1_{s=t}$ where 1 . is the indicator function, and define the linear process $u_{t}=\mu_{t}+C(L) \varepsilon_{t}$, where $\mu_{t}$ is a nonstochastic $p \times 1$ vector and $C(z)=\sum_{j=0}^{\infty} z^{j} C_{j}^{\circ}$ is a $p \times p$ matrix function, with coefficient matrices $C_{j}^{\circ} \in \mathbb{R}^{p \times p}$. Note that the matrices $C_{j}^{\circ}$ are defined by an expansion of $C(z)$ around $z=0$. The term $\mu_{t}$ is nonstochastic, i.e., $\mathrm{E}\left(\mu_{t}\right)=\mu_{t}$, and can contain deterministic terms. Because $\mathrm{E}\left(\varepsilon_{t}\right)=0$, one sees that $\mathrm{E}\left(u_{t}\right)=\mu_{t}$, and hence in the following $u_{t}$ is often written as $u_{t}=\mathrm{E}\left(u_{t}\right)+C(L) \varepsilon_{t}$.

The matrix function $C(z)=\sum_{j=0}^{\infty} z^{j} C_{j}^{\circ}$ is assumed to be finite when $z$ is inside the open disk $D(0,1+\eta), \eta>0$, in $\mathbb{C}$ with center at 0 and radius $1+\eta>1$, i.e., $C(z)$ is assumed analytic on $D(0,1+\eta)$. Here and in the following $|\cdot|$ indicates the modulus and $D\left(z_{\star}, \rho\right)$ indicates the open disk $D\left(z_{\star}, \rho\right):=\left\{z \in \mathbb{C}:\left|z-z_{\star}\right|<\rho\right\}$ with center $z_{\star} \in \mathbb{C}$ and radius $\rho>0$. In this paper $C(z)$ is assumed to be regular on $D(0,1+\eta)$, i.e., $C(z)$ can lose rank only at a finite number of isolated points in $D(0,1+\eta)$.

Because of analyticity of $C(z)$, it can be expanded around any interior point of $D(0,1+\eta)$. In particular, define the point $z_{\omega}:=e^{i \omega}$ on the unit circle at frequency $\omega, \omega \in[0,2 \pi)$, and observe that it lies inside $D(0,1+\eta)$ because $\eta>0$. Hence one can expand $C(z)$ as $C(z)=\sum_{j=0}^{\infty}\left(z-z_{\omega}\right)^{j} C_{j}$ on $D\left(z_{\omega}, \eta\right), \eta>0$. Note that the matrices $C_{j}$ are defined by an expansion of $C(z)$ around $z=z_{\omega}$, but that the dependence of $C_{j}$ on $\omega$ is not included in the notation for simplicity. The analysis of the properties of $C(z)$ is done locally around $z=z_{\omega}$ on $D\left(z_{\omega}, \eta\right), \eta>0$.

Similarly to $C(z)$, one can consider a scalar function of $z, a(z)$ say, or a $1 \times p$ vector function $b(z)^{\prime}$ taken to be analytic on $D\left(z_{\omega}, \eta\right), \eta>0$. This means that $a(z)$ has representation $a(z)=\sum_{j=0}^{\infty}\left(z-z_{\omega}\right)^{j} a_{j}$ around $z_{\omega}$ and similarly for $b(z)^{\prime}$. A special case is when $a(z)$ is a polynomial of degree $k, a(z)=\sum_{j=0}^{k}\left(z-z_{\omega}\right)^{j} a_{j}$, which corresponds to setting all $a_{j}=0$ for $j>k$. Another special case is given by rational functions $a(z)=c(z) / d(z)$ with $c(z)$ and $d(z)$ polynomials, where $d(z) \not \equiv 0$ and $z_{\omega}$ is not a root of $d(z)$. Similarly for $b(z)^{\prime}$.

\subsection{Integration}

The following definition specifies the $I_{\omega}(0)$ class of processes as a subset of all linear processes built from the noise sequence $\varepsilon_{t}$, and introduces the notion of $I_{\omega}(d)$ processes using the difference operator at frequency $\omega, \Delta_{\omega}:=1-e^{-i \omega} L=1-z_{\omega}^{-1} L$. To simplify notation, the dependence of $\Delta_{\omega}$ on the lag operator $L$ is left implicit. Observe also that, because $z_{\omega}=e^{i \omega} \neq 0, z-z_{\omega}$ in the analytic expansions can be expressed as $\left(-z_{\omega}\right)(1-$ $\left.z / z_{\omega}\right)$, where $\left(1-z / z_{\omega}\right)$ corresponds to the operator $\Delta_{\omega}$.

Next, the definition of order of integration is introduced; this is defined as the difference between two nonnegative integer exponents $d_{1}$ and $d_{2}$ of $\Delta_{\omega}$ in the representation that links the process $x_{t}$ with its driving linear process $u_{t}$. This definition allows for the possibility to have $x_{t}$ integrated of negative order.

Definition 1 (Integrated processes at frequency $\omega$ ). Let $C(z)$ be analytic on $D(0,1+\eta)$, $\eta>0$, and let $\varepsilon_{t}$ be a noise process. If $\left\{u_{t}, t \in \mathbb{Z}\right\}$, satisfies $u_{t}=\mathrm{E}\left(u_{t}\right)+C(L) \varepsilon_{t}$, then $u_{t}$ is called a linear process; if, in addition,

$$
C\left(z_{\omega}\right) \neq 0, \quad z_{\omega}=\exp (i \omega), \quad 0 \leq \omega<2 \pi,
$$


then $u_{t}$ is said to be integrated of order zero at frequency $\omega$, indicated $u_{t} \sim I_{\omega}(0)$.

Let $d_{1}, d_{2} \in \mathbb{N}_{0}=\mathbb{N} \cup 0$ be finite non-negative integers; if $\left\{x_{t}, t \in \mathbb{Z}\right\}$ satisfies $\Delta_{\omega}^{d_{1}}\left(x_{t}-\right.$ $\left.\mathrm{E}\left(x_{t}\right)\right)=\Delta_{\omega}^{d_{2}}\left(u_{t}-\mathrm{E}\left(u_{t}\right)\right)$ where $u_{t} \sim I_{\omega}(0)$, then $x_{t}$ is said to be integrated of order $d:=d_{1}-d_{2}$ at frequency $\omega$, indicated $x_{t} \sim I_{\omega}(d)$; in this case $x_{t}$ has representation

$$
\Delta_{\omega}^{d_{1}}\left(x_{t}-\mathrm{E}\left(x_{t}\right)\right)=\Delta_{\omega}^{d_{2}} \mathrm{C}(L) \varepsilon_{t}
$$

where $C(z)$ is analytic on $D(0,1+\eta), \eta>0$, and $C\left(z_{\omega}\right) \neq 0$.

Remark 3 (Negative orders). When $d_{1}<d_{2}$, the integration order $d:=d_{1}-d_{2}$ is negative. Note also that Definition 1 avoids to define the operator $\Delta_{\omega}^{-1}$; see however Equations (5) and (6) below.

Remark 4 (Mean-0 linear process). The linear process $u_{t}$ in Definition 1 can have any expectation $\mathrm{E}\left(u_{t}\right)$, which however, does not play any role in the definition of the $x_{t}$ process. Hence, one can assume that $\mathrm{E}\left(u_{t}\right)=0$ in Definition 1 without loss of generality.

Remark $5\left(\mathrm{E}\left(x_{t}\right)\right.$ in Definition 1). Assume $x_{t}=\cos (2 t)+\exp (-3 t)+C(L) \varepsilon_{t}$ with $C(z)$ analytic on $D(0,1+\eta), \eta>0$, and $C\left(z_{\omega}\right) \neq 0, z_{\omega}:=e^{i \omega}$. Then $\mathrm{E}\left(x_{t}\right)=\cos (2 t)+\exp (-3 t)$ and Definition 1 implies that $x_{t}$ is $I_{\omega}(0)$. This example shows that the presence of $\mathrm{E}\left(x_{t}\right)$ in Equation (2) allows to concentrate attention on the stochastic part of the process $x_{t}$.

Remark 6 (Preference for low $\left.d_{1}, d_{2}\right)$. Assume for instance that (2) is satisfied for $\left(d_{1}, d_{2}\right)=$ $(1,0)$, and observe that this implies that (2) is satisfied for $\left(d_{1}, d_{2}\right)=(1+m, m)$ for any $m \in \mathbb{N}$. In the following, preference is given to the minimal pair $\left(d_{1}, d_{2}\right)$ for which (2) is satisfied, i.e., to $\left(d_{1}, d_{2}\right)=(1,0)$ in the example.

Leading cases are the ones where either $d_{1}$ or $d_{2}$ equals 0 . Specifically, when $0=d_{1}<d_{2}$, $d=d_{1}-d_{2}=-d_{2}$ is negative, and (2) reads

$$
x_{t}-\mathrm{E}\left(x_{t}\right)=\Delta_{\omega}^{d_{2}} C(L) \varepsilon_{t} .
$$

When $d_{1} \geq d_{2}=0$ and hence $d=d_{1}-d_{2}=d_{1}$ is nonnegative, (2) reads

$$
\Delta_{\omega}^{d_{1}}\left(x_{t}-\mathrm{E}\left(x_{t}\right)\right)=C(L) \varepsilon_{t}
$$

Remark 7 (Example of $I_{0}(-1)$ ). As an example, consider the process $x_{t}=C(L) \varepsilon_{t}$ with $C(L)=$ $1-$ L. Setting $\omega=0$ one finds that Equation (2) is satisfied with $d=-1$, i.e., that the process is $I_{0}(-1)$. Selecting any other frequency $0<\omega<2 \pi$, one sees that Equation (2) is satisfied for $d=0$, i.e., that the order of integration is 0 , i.e., $I_{\omega}(0)$ for $0<\omega<2 \pi$. This illustrates the fact that a process may have different orders of integration at different frequencies.

Remark $8\left(t \in \mathbb{Z}\right.$ versus $\left.t \in \mathbb{N}_{0}\right)$. Consider the process $x_{t}=c+\sum_{j=1}^{t} \varepsilon_{t}$ defined only for $t \in \mathbb{N}_{0}=\mathbb{N} \cup 0$, which satisfies $\Delta_{0}\left(x_{t}-c\right)=\varepsilon_{t}$ for $t \in \mathbb{N}$. Consider another process $\left\{x_{t}^{\star}, t \in \mathbb{Z}\right\}$ satisfying the same equation $\Delta_{0}\left(x_{t}^{\star}-c\right)=\varepsilon_{t}$ for $t \in \mathbb{Z}$ with $x_{t}=x_{t}^{\star}$ for $t \in \mathbb{N}_{0}$. The process $\left\{x_{t}^{\star}, t \in \mathbb{Z}\right\}$ is $I_{0}(1)$ according to Definition 1 , and it is suggested to extend this qualification to $x_{t}$, because it coincides with the $x_{t}^{\star}$ process on the non-negative integers, $x_{t}=x_{t}^{\star}$ for $t \in \mathbb{N}_{0}$.

Remark 9 (One or more frequencies). Definition 1 of integration refers to a single frequency $\omega$, but it can be used to cover multiple frequencies. In fact, consider the 'ARMA process with unit root structure', as defined in Bauer and Wagner (2012), i.e., a process $x_{t}$ satisfying $D(L) x_{t}=v_{t}$ where $D(L):=\prod_{j=1}^{n} \Delta_{\omega_{j}}^{m_{i}}$ for a (finite) set of frequencies $\omega_{1}, \ldots, \omega_{n}$, with $v_{t}$ a stationary ARMA process $v_{t}=C(L) \varepsilon_{t}$ with $C\left(\exp \left(i \omega_{j}\right)\right) \neq 0$. They call $\left\{\left(\omega_{j}, m_{j}\right), j=1, \ldots, n\right\}$, the 'unit root structure' of $x_{t}$, see their Definition 2. This can be obtained using Definition 1 for each $\omega_{j}$ in turn, noting that $v_{t}$ being ARMA corresponds to a rational $C(z)$, which is a special case of the definition above. 
Hylleberg et al. (1990), Gregoir (1999), Johansen and Schaumburg (1998), Bauer and Wagner (2012) consider $x_{t}$ to be real-valued, which implies that integration frequencies $\pm \omega_{j}$ are 'paired', so that if $\exp \left(i \omega_{j}\right)$ is a unit root of the process, so is $\exp \left(-i \omega_{j}\right)$; this implies that in this case one can pair frequencies $\pm \omega_{j}$ with $0<\omega_{j}<\pi$ and rearrange coefficients so as to obtain real coefficient matrices in EC representations. This is not done in this paper for reasons of simplicity.

Remark 10 (Relation with other definitions). The definition of an $I_{\omega}(0)$ (respectively an $I_{\omega}(d)$ ) process in the present Definition 1 coincides with Definition 3.2 (respectively Definition 3.3) in Johansen (1996) when setting $\omega=0$ (respectively $\omega=0$ and $d_{2}=0$ ). The present definition also agrees with Definitions 2.1 and 2.2 of integration in Gregoir (1999), both for positive and negative orders and any frequency $\omega$. The definition also agrees with the one in Franchi and Paruolo (2019) when applied to vector processes.

Remark 11 (Entries in $C(z)$ ). When $\omega$ differs from 0 or $\pi$, the point $z_{\omega}=e^{i \omega}$ has a nonzero complex part; hence the matrix $C\left(z_{\omega}\right)$ in (1) has complex entries and the coefficient matrices $C_{j}$ in the expansion $C(z)=\sum_{j=0}^{\infty}\left(z-z_{\omega}\right)^{j} C_{j}$ are complex even when the coefficients in the expansion around $z=0$ are real.

Following Gregoir (1999), the summation operator at frequency $\omega$ is defined as

$$
\mathcal{S}_{\omega} u_{t}:=1_{t>0} \sum_{j=1}^{t} u_{j} e^{-i \omega(t-j)}-1_{t<0} \sum_{j=t+1}^{0} u_{j} e^{-i \omega(t-j)} .
$$

Basic properties of the operator are proved in Gregoir (1999); these include

$$
\Delta_{\omega} \mathcal{S}_{\omega} u_{t}=u_{t}, \quad \mathcal{S}_{\omega} \Delta_{\omega} u_{t}=u_{t}-u_{0} e^{-i \omega t}
$$

where $\left\{u_{t}, t \in \mathbb{Z}\right\}$ is any sequence over $\mathbb{Z}$.

Remark 12 (Simplifications of $\Delta_{\omega}$ and initial values). Take $d_{1}=d_{2}=1$ in (2), which in this case reads $\Delta_{\omega} x_{t}=\Delta_{\omega} u_{t}$ with $u_{t} \sim I_{\omega}(0)$. Applying the $\mathcal{S}_{\omega}$ operator on both sides one obtains $x_{t}-x_{0} e^{-i \omega t}=u_{t}-u_{0} e^{-i \omega t} \cdot{ }^{4}$ If one assigns the initial value of $x_{0}$ equal to $u_{0}$, one obtains $x_{t}=u_{t}$, which corresponds to the cancellation of $\Delta_{\omega}$ from both sides of (2). The same reasoning applies for generic $d_{1}, d_{2}>0$ to the cancellation of $\Delta_{\omega}^{\min \left(d_{1}, d_{2}\right)}$ from both sides of (2). This shows that one can simplify powers of $\Delta_{\omega}$ from both sides of (2) by properly assigning initial values; this cancellation is always implicitly performed in the following, in line with preference for minimal values of $d_{1}, d_{2}$ as discussed in Remark 6.

\subsection{Cointegration}

Cointegration is the property of (possibly polynomial) linear combinations of $x_{t}$ to have a lower order of integration with respect to the original order of integration of $x_{t}$ at frequency $\omega$. Specifically, consider a nonzero $1 \times p$ row vector function $\zeta(z)^{\prime}=$ $\sum_{j=0}^{\infty} \zeta_{j}^{\prime}\left(z-z_{\omega}\right)^{j}$, analytic on a disk $D\left(z_{\omega}, \eta\right), \eta>0$. As in Engle and Granger (1987), the idea is to call $\zeta(L)^{\prime}$ cointegrating if $\zeta(L)^{\prime} x_{t}$ has lower order of integration than $x_{t}$, excluding cases such as $\zeta(L)^{\prime}=\Delta_{\omega} a^{\prime}$ where $a^{\prime}$ by itself does not reduce the order of integration.

This leads to the following definition.

Definition 2 (Cointegrating vector at frequency $\omega$ ). Let $x_{t} \sim I_{\omega}(d)$ be as in Definition 1, i.e.,

$$
\Delta_{\omega}^{d_{1}}\left(x_{t}-\mathrm{E}\left(x_{t}\right)\right)=\Delta_{\omega}^{d_{2}} \mathrm{C}(L) \varepsilon_{t}
$$

where $d:=d_{1}-d_{2}, C(z)$ is analytic on $D(0,1+\eta), \eta>0$, and $C\left(z_{\omega}\right) \neq 0$, see (2); let also $\zeta(z)^{\prime}=\sum_{j=0}^{\infty}\left(z-z_{\omega}\right)^{j} \zeta_{j}^{\prime}$ be a $1 \times p$ row vector function, analytic on $D\left(z_{\omega}, \eta\right)$ with $\zeta\left(z_{\omega}\right)^{\prime}=$ 
$\zeta_{0}^{\prime} \neq 0^{\prime}$. Then $\zeta(z)^{\prime}$ is called a cointegrating vector at frequency $\omega$ if $\zeta(L)^{\prime} x_{t} \sim I_{\omega}(d-s)$ for some $s \in \mathbb{N}$, i.e.,

$$
\zeta(L)^{\prime} \Delta_{\omega}^{d_{1}}\left(x_{t}-\mathrm{E}\left(x_{t}\right)\right)=\Delta_{\omega}^{d_{2}+s} g(L)^{\prime} \varepsilon_{t}
$$

where $g(z)^{\prime}$ is analytic on $D\left(z_{\omega}, \eta\right), \eta>0$, and $g\left(z_{\omega}\right)^{\prime} \neq 0^{\prime}$. Given Equation (2), Equation (7) is equivalent to the condition

$$
\zeta(L)^{\prime} C(L)=\Delta_{\omega}^{s} g(L)^{\prime}, \quad g\left(z_{\omega}\right)^{\prime} \neq 0^{\prime} .
$$

The positive integer $s \in \mathbb{N}$ is called the order of the cointegrating vector $\zeta(z)^{\prime}$ of $C(z)$ at $z_{\omega}$. $x_{t}$ is said to be cointegrated at frequency $\omega$ if any cointegrating vector $\zeta(z)^{\prime}=\sum_{j=0}^{\infty}\left(z-z_{\omega}\right)^{j} \zeta_{j}^{\prime}$ can be replaced by $\zeta\left(z_{\omega}\right)^{\prime}=\zeta_{0}^{\prime}$ without decreasing the order $s$ in (8); otherwise $x_{t}$ is said to be multicointegrated at frequency $\omega$.

Remark $13\left(C(z)\right.$ has full rank on $D\left(z_{\omega}, \eta\right), \eta>0$, except at $\left.z=z_{\omega}\right)$. Because cointegrating vectors are by definition different from zero at $z_{\omega}, x_{t}$ is cointegrated at frequency $\omega$ if and only if $C\left(z_{\omega}\right) \neq 0$ has reduced rank. Moreover, because $C(z)$ is regular on $D(0,1+\eta)$, the point $z_{\omega}$ is isolated, i.e., $C(z)$ has full rank on $D\left(z_{\omega}, \eta\right), \eta>0$, except at $z=z_{\omega}$.

Remark 14 (Entries in cointegrating vectors). Similarly to Remark 11, the coefficient vectors $\zeta_{j}^{\prime}$ in the expansion $\zeta(z)^{\prime}=\sum_{j=0}^{\infty}\left(z-z_{\omega}\right)^{j} \zeta_{j}^{\prime}$ are in general complex. Note that $\zeta(L)^{\prime}=\Delta_{\omega} a^{\prime}$ does not satisfy the definition because the requirement $\zeta\left(z_{\omega}\right)^{\prime}=\zeta_{0}^{\prime} \neq 0^{\prime}$ is not satisfied.

Remark 15 ( $d$ and $s)$. Recall that $d$ (the order of integration) is the difference between the exponents of $\Delta_{\omega}$ on the l.h.s. and r.h.s. of (2). When pre-multiplied by $\zeta(L)^{\prime}$, the exponent on the r.h.s. decreases by $s$ and the difference of the exponents on the l.h.s. and r.h.s. of (7) becomes $d-s$. Because $\zeta_{0}^{\prime} \neq 0^{\prime}$, this can only happen if $\zeta(L)^{\prime}$ factors $\Delta_{\omega}^{s}$ from $C(L)$, see (8). The condition $g\left(z_{\omega}\right)^{\prime} \neq 0^{\prime}$ guarantees that no remaining additional power of $\Delta_{\omega}$ can be factored from $C(L)$ using $\zeta(L)^{\prime}$.

Remark 16 (Examples of cointegration vectors). Take $\zeta(L)^{\prime}=\zeta_{0}^{\prime}$ with $\zeta_{0}$ chosen in $(\operatorname{col} C(1))^{\perp}$, and note that this implies $s \geq 1$ in (7). This shows that the definition contains the $I_{0}(1)$ definition of cointegrating vectors as a special case.

The usual definition of cointegration, see Definition 3.4 in Johansen (1996), considers a $p \times 1$ process $x_{t} \sim I_{0}(1)$ and defines $x_{t}$ cointegrated with cointegrating vector $\zeta \neq 0$ if $\zeta^{\prime} x_{t}$ "can be made stationary by a suitable choice of initial distribution". The following proposition clarifies that his definition coincides with the one in this paper.

Proposition 1 (Relation with Definition 3.4 in Johansen (1996)). $\zeta^{\prime}$ is a cointegrating vector in the sense of Definition 3.4 in Johansen (1996) if and only if Definition 2 is satisfied with $\omega=0$ and $\zeta(z)^{\prime}=\zeta^{\prime}, d=1, s \in \mathbb{N}$.

Proof. For simplicity and without loss of generality, set $\mathrm{E}\left(x_{t}\right)=0$ and omit the subscript $\omega=0$. Assume Definition 2 is satisfied with $\omega=0$ and $\zeta(z)^{\prime}=\zeta^{\prime}, d=1$, and $s \in \mathbb{N}$, i.e.,

$$
\Delta \zeta^{\prime} x_{t}=\Delta^{s} g(L) \varepsilon_{t}
$$

see Remark 12, and set $v_{t}:=\Delta^{s-1} g(L) \varepsilon_{t}$. Applying $\mathcal{S}$ to both sides of Equation (9) one finds $\zeta^{\prime} x_{t}-\zeta^{\prime} x_{0}=v_{t}-v_{0}$. Note that $v_{t}$ is stationary for any $s \in \mathbb{N}$, and hence the initial values $\zeta^{\prime} x_{0}$ can be chosen equal to $v_{0}$, so as to obtain $\zeta^{\prime} x_{t}=v_{t}$, a stationary process.

Conversely, assume that $\zeta^{\prime}$ is a cointegrating vector in the sense of Definition 3.4 in Johansen (1996). Because $x_{t} \sim I(1)$, one has $\Delta x_{t}=C(L) \varepsilon_{t}$, see Definition 1 , with $C(z)$ analytic on a disk $D(0,1+\eta), \eta>0$, which admits expansion $C(z)=C+\widetilde{C}(z)(1-z)$ around 1 , where $\widetilde{C}(z)$ is analytic on the same disc. A necessary and sufficient condition for 
cointegration in the sense of Definition 3.4 in Johansen (1996) is that $\zeta^{\prime} C=0$ as shown in Johansen (1988) Equation (17); see also Engle and Granger (1987, p. 256). ${ }^{5}$ Hence one finds $\zeta^{\prime} \Delta x_{t}=\Delta g(L)^{\prime} \varepsilon_{t}$ with $g(z):=\zeta^{\prime} \widetilde{C}(z)$, which is analytic on $D(0,1+\eta), \eta>0$, and hence also on $D(1, \eta), \eta>0$. By Corollary 1 below, one has that $g(z)^{\prime}$ satisfies $g(z)^{\prime}=\Delta^{m} \widetilde{g}(z)^{\prime}$ with finite $m \in \mathbb{N}_{0}$ and $\widetilde{g}\left(z_{\omega}\right)^{\prime} \neq 0^{\prime}$. This shows that Definition 2 is satisfied with $\zeta(z)^{\prime}=\zeta^{\prime}$, $d=1$, and $s=m+1 \in \mathbb{N}$.

Remark 17 ( $\zeta^{\prime} x_{t}$ can have negative order of integration). Johansen (1996) makes the following observation just after his Definition 3.4: "Note that $\zeta^{\prime} x_{t}$ need not be $I(0)$ ", which recognises that $\zeta^{\prime} x_{t}$ can have negative order of integration. This is indeed the case when to $s=2,3, \ldots$ in Definition 2 , because $\zeta^{\prime} x_{t} \sim I(1-s)$.

Remark 18 (Relation to other definitions in the literature). The definition of cointegration in Engle and Granger (1987) reported in the introduction is a special case of the present one with $\zeta(z)^{\prime}=\zeta_{0}^{\prime}$ a constant vector and $\omega=0$, under the additional requirement that all variables are integrated of the same order. For more details on this for the case $\omega=0$, see Franchi and Paruolo (2019). When $s>1$ and $\omega=0$, Definition 2 covers the definitions of multicointegration and polynomial cointegration in Granger and Lee (1989), Engle and Yoo (1991), Johansen (1996). When s = 1 and $\omega=2 \pi j / n$ for $j=1, \ldots, n$ where $n$ is the number of seasons, the definition covers seasonal cointegration in Hylleberg et al. (1990), Johansen and Schaumburg (1998).

Example 1 (I(1) VAR). Following Johansen (1988), consider $A(L) x_{t}=\varepsilon_{t}$ with $A(z)=I-$ $\sum_{j=1}^{k}(1-z)^{j} A_{j}$ analytic on $\mathbb{C}$. Assume also that $\operatorname{det} A(z)=0$ has only solutions outside $D(0,1+$ $\eta), \eta>0$, or at $z=1$, where 'det' indicates the determinant of a matrix. Here and in the following, let $a_{\perp}$ indicate $a$ basis of the orthogonal complement of the linear space spanned by the columns of the matrix $a$. Moreover $P_{a}:=a\left(a^{\prime} a\right)^{-1} a^{\prime}$ for a full-column-rank matrix $a$ is the orthogonal projection matrix onto $\operatorname{col}(a)$. Johansen (1991) (see his Equations (4.3) and (4.4) in Theorem 4.1) showed that for $x_{t}$ to be $I(1)$ at frequency $\omega=0$, a set of necessary and sufficient conditions are:

(i) $A(1)=-\alpha_{0} \beta_{0}^{\prime}$ with $\alpha_{0}, \beta_{0}$ full column rank matrices of dimension $p \times r_{0}, r_{0}<p$,

(ii) $P_{\alpha_{0 \perp}} A_{1} P_{\beta_{0 \perp}}=-\alpha_{1} \beta_{1}^{\prime}$ of maximal rank $r_{1}=p-r_{0}$.

In this case $x_{t}$ satisfies (2) for $d_{1}=1, d_{2}=0$, and $\zeta(L)^{\prime}=\zeta^{\prime}$ taken to be any row vector in $\mathcal{B}=\operatorname{row}_{F}\left(\beta_{0}^{\prime}\right)$ with $F=\mathbb{R}$.

Example 2 (I(2) VAR). Following Johansen (1992), consider the same VAR process as in Example 1. Johansen (1992) showed that for $x_{t}$ to be I(2) at frequency $\omega=0$, a set of necessary and sufficient conditions are:

(i) $A(1)=-\alpha_{0} \beta_{0}^{\prime}$ with $\alpha_{0}, \beta_{0}$ full column rank matrices of dimension $p \times r_{0}, r_{0}<p$,

(ii) $P_{\alpha_{0 \perp}} A_{1} P_{\beta_{0 \perp}}=-\alpha_{1} \beta_{1}^{\prime}$ with $\alpha_{1}, \beta_{1}$ full column rank matrices of dimension $p \times r_{1}, r_{1}<p-r_{0}$,

(iii) $P_{\left(\alpha_{0}, \alpha_{1}\right)_{\perp}}\left(A_{2}+A_{1} \bar{\beta}_{0} \bar{\alpha}_{0}^{\prime} A_{1}\right) P_{\left(\beta_{0}, \beta_{1}\right)_{\perp}}=-\alpha_{2} \beta_{2}^{\prime}$ of maximal rank $r_{2}=p-r_{0}-r_{1}$.

In this case $x_{t}$ satisfies (2) for $d_{1}=2, d_{2}=0$, and $\zeta(L)^{\prime}=\zeta_{0}^{\prime}+\Delta \zeta_{1}^{\prime}$ taken to be any row vector obtained by linear combinations of the rows in $\beta_{0}^{\prime}+(1-L) \bar{\alpha}_{0}^{\prime} A_{1}$ and $\beta_{1}^{\prime}$. The notion of cointegrating space for I(2) processes is discussed in detail below, where $\bar{\alpha}_{0}^{\prime} A_{1}$ is called the 'multicointegrating coefficient'.

\section{Root Functions, Cointegrating Vectors and Canonical Systems}

This section introduces root functions and canonical systems of root functions, and their connection to cointegrating vectors, as defined in Definition 2 above.

\subsection{Root Functions}

Let $x_{t} \sim I_{\omega}(d)$ be cointegrated at frequency $\omega$, i.e., see Definition 2,

$$
\Delta_{\omega}^{d_{1}}\left(x_{t}-\mathrm{E}\left(x_{t}\right)\right)=\Delta_{\omega}^{d_{2}} C(L) \varepsilon_{t},
$$


where $d:=d_{1}-d_{2}$ and $C(z)$ has full rank on $D\left(z_{\omega}, \eta\right), \eta>0$, except at $z=z_{\omega}$, see Remark 13.

The following definition of (left) root functions is taken from Gohberg et al. (1993); this definition is given in a neighborhood of $z_{\omega}$.

Definition 3 (Root function). $A 1 \times p$ row vector function $\varphi(z)^{\prime}$ analytic on $D\left(z_{\omega}, \eta\right)$ is called a root function of $C(z)$ at $z_{\omega}$ if $\varphi\left(z_{\omega}\right)^{\prime} \neq 0^{\prime}$ and if

$$
\varphi(z)^{\prime} C(z)=\left(z-z_{\omega}\right)^{s} \widetilde{\varphi}(z)^{\prime}, \quad s \in \mathbb{N}, \quad \widetilde{\varphi}\left(z_{\omega}\right)^{\prime} \neq 0^{\prime} .
$$

The positive integer s is called the order of the root function $\varphi(z)^{\prime}$ at $z_{\omega}$.

Observe that $\widetilde{\varphi}(z)^{\prime}$ is $1 \times p$ and analytic on $D\left(z_{\omega}, \eta\right), \eta>0$.

Remark 19 (Factoring the difference operator). Definition 3 characterizes roots functions by their ability to factor powers of $\left(z-z_{\omega}\right)$ from $C(z)$. Note that, because here $z_{\omega}=\exp (i \omega) \neq 0$, one can write $\left(z-z_{\omega}\right)$ as $\left(-z_{\omega}\right)\left(1-z / z_{\omega}\right)$ where $\left(1-z / z_{\omega}\right)$ corresponds to the difference operator $\Delta_{\omega}$ and $\left(-z_{\omega}\right)$ can be absorbed in $\widetilde{\varphi}(z)^{\prime}$ without affecting its property that $\widetilde{\varphi}\left(z_{\omega}\right)^{\prime} \neq 0^{\prime}$.

Remark 20 (Local analysis). Note first that $C(z)$ cannot be identically 0 in Definition 3, because $C(z)$ is assumed to be regular. Next take for example the $2 \times 2$ matrix $C(z)=\operatorname{diag}((1-z),(1+z))$ which has full rank on $\mathbb{C}$, except at the two points $z_{0}=1$ and $z_{\pi}=-1$, where it has rank 1 .

Take first the point at $z_{0}=1$; in this case one could choose a disk $D(1, \eta)$ with any $\eta<2$, on which $C(z)$ is analytic and full rank except at $z_{0}=1$. One can verify that a root function is $\varphi_{1}(z)^{\prime}=(1,0)$, which satisfies $\varphi_{1}(z)^{\prime} C(z)=(1-z) \widetilde{\varphi}_{1}(z)^{\prime}$ with $\widetilde{\varphi}_{1}(z)^{\prime}=(1,0)$. The same can be repeated for the other point $z_{\pi}=-1$, choosing a different disk $D(-1, \eta)$ with any $\eta<2$, and a root function equal to $(0,1)$.

The implication of this example is that one can have multiple separated points where $C(z)$ has reduced rank, and apply the above definition to each point separately, using a different disk $D$ for each point. In other words, the discussion of cointegration in this paper is local to a single unit root.

Remark 21 (Order). A root function factorises $\left(z-z_{\omega}\right)^{s}$ from $C(z)$, and s indicates the order. The condition $\varphi\left(z_{\omega}\right)^{\prime} \neq 0^{\prime}$ guarantees that in the analytic expansion $\varphi(z)^{\prime}=\sum_{n=0}^{\infty}\left(z-z_{\omega}\right)^{n} \varphi_{n}^{\prime}$, the first term $\varphi_{0}^{\prime}$ is not the null vector. Note that the condition $\widetilde{\varphi}\left(z_{\omega}\right)^{\prime} \neq 0^{\prime}$ makes sure that one cannot extract additional factors of $\left(z-z_{\omega}\right)$ from $C(z)$ using $\varphi(z)^{\prime}$.

It is immediate to see that a cointegrating vector is a root function of $C(z)$ and vice versa, as stated in the following theorem.

Theorem 1 (Cointegrating vectors and root functions). $\zeta(z)^{\prime}$ is a cointegrating vector at frequency $\omega$ if and only if $\zeta(z)^{\prime}$ is a root function of $C(z)$ at $z_{\omega}$, and the order of the cointegrating vector and of the root function coincide.

Proof. Observe that any root function satisfies Definition 2 of cointegrating vectors and vice versa, including the definition of their order.

Results in Gohberg et al. (1993) shows that the order of a root functions is finite, because it is bounded by the order of $z_{\omega}$ as a zero of $\operatorname{det} C(z)$, a result that is reported in the next proposition.

Proposition 2 (Bound on the order of a root function). The order of a root function of $C(z)$ at $z_{\omega}$ is at most equal to the order of $z_{\omega}$ as a zero of $\operatorname{det} C(z)$, which is finite because $C(z)$ is regular.

Proof. See Gohberg et al. (1993). 
Corollary 1 (Bound on the order of a cointegrating vector). The order of any cointegrating vector at frequency $\omega$ is finite.

Proof. This follows from Proposition 2 because cointegrating vectors and root functions coincide by Theorem 1 .

\subsection{Canonical Systems of Root Functions}

Next, canonical systems of root functions for $C(z)$ at $z_{\omega}$ are introduced, see Gohberg et al. (1993). Choose a root function $\phi_{1}(z)^{\prime}$ of highest order $s_{1}$. Since the orders of the root functions are bounded by Proposition 2, such a function exists. Next proceed iteratively over $j=2, \ldots$, choosing the next root function $\phi_{j}(z)^{\prime}$ to be of the highest order $s_{j}$ such that $\phi_{j}\left(z_{\omega}\right)^{\prime}$ is linearly independent from $\phi_{1}\left(z_{\omega}\right)^{\prime}, \ldots, \phi_{j-1}\left(z_{\omega}\right)^{\prime}$. Because $m:=\operatorname{dim}\left(\left(\operatorname{col} C\left(z_{\omega}\right)\right)^{\perp}\right)<\infty$, this process ends with $m$ root functions $\phi_{1}(z)^{\prime}, \ldots, \phi_{m}(z)^{\prime}$.

Note that the columns in $a:=\left(\phi_{1}\left(z_{\omega}\right), \ldots, \phi_{m}\left(z_{\omega}\right)\right)$ span the finite dimensional space $\left(\operatorname{col} C\left(z_{\omega}\right)\right)^{\perp}$, so that one can choose vectors $\left(\phi_{m+1}, \ldots, \phi_{p}\right)=a_{\perp}$ that span its orthogonal complement. This construction leads to the following definition.

Definition 4 ((Extended) canonical system of root functions). Let $\phi_{1}(z)^{\prime}, \ldots, \phi_{m}(z)^{\prime}$ and $\phi_{m+1}^{\prime}, \ldots, \phi_{p}^{\prime}$ be constructed as above; then

$$
\phi(z)^{\prime}=\left(\begin{array}{c}
\phi_{1}(z)^{\prime} \\
\vdots \\
\phi_{m}(z)^{\prime}
\end{array}\right) \quad \text { and } \quad\left(\begin{array}{c}
\phi(z)^{\prime} \\
\hdashline a_{\perp}^{\prime}
\end{array}\right)=\left(\begin{array}{c}
\phi_{1}(z)^{\prime} \\
\vdots \\
\phi_{m}(z)^{\prime} \\
\hdashline \phi_{m+1}^{\prime--} \\
\vdots \\
\phi_{p}^{\prime}
\end{array}\right)
$$

are called a canonical system of root functions (respectively an extended canonical system of root functions) of $C(z)$ at $z_{\omega}$ of orders $\left(s_{1}, s_{2}, \ldots, s_{m}\right)$ (respectively $\left(s_{1}, s_{2}, \ldots, s_{m}, s_{m+1}, \ldots, s_{p}\right)$ ) with $\infty>s_{1} \geq s_{2} \geq \cdots \geq s_{m}>0=s_{m+1}=\cdots=s_{p}$.

Such a canonical system of root functions is not unique. To see this, one can show that the first row vector $\phi_{1}(z)^{\prime}$ in (11) can be replaced by a combination of $\phi_{1}(z)^{\prime}$ and $\phi_{2}(z)^{\prime}$, called $\phi_{1}^{\star}(z)^{\prime}$, and the canonical system of root functions containing $\phi_{1}^{\star}(z)^{\prime}$ would still satisfy the definition. More specifically, define $\phi_{1}^{\star}(z)^{\prime}:=\phi_{1}(z)^{\prime}+\left(z-z_{\omega}\right)^{s_{1}-s_{2}} \phi_{2}(z)^{\prime}$ and observe that, by Definition 3, $\phi_{j}(z)^{\prime} C(z)=\left(z-z_{\omega}\right)^{s_{j}} \widetilde{\phi}_{j}(z)^{\prime}$, with $\widetilde{\phi}_{j}\left(z_{\omega}\right)^{\prime} \neq 0^{\prime}, j=1,2$. Hence one has

$$
\phi_{1}^{\star}(z)^{\prime} C(z)=\left(z-z_{\omega}\right)^{s_{1}} \widetilde{\phi}_{1}^{\prime}(z)+\left(z-z_{\omega}\right)^{s_{1}-s_{2}+s_{2}} \widetilde{\phi}_{2}^{\prime}(z)=\left(z-z_{\omega}\right)^{s_{1}} \widetilde{\phi}^{\star \prime}(z)
$$

where $\widetilde{\phi}^{\prime \star}(z):=\widetilde{\phi}_{1}^{\prime}(z)+\widetilde{\phi}_{2}^{\prime}(z)$. Because $\widetilde{\phi}_{j}\left(z_{\omega}\right)^{\prime} \neq 0^{\prime}, j=1,2$, one has $\widetilde{\phi}^{\prime \star}\left(z_{\omega}\right) \neq 0^{\prime}$ unless $\widetilde{\phi}_{1}\left(z_{\omega}\right)^{\prime}=-\widetilde{\phi}_{2}\left(z_{\omega}\right)^{\prime}$. However, this last case is ruled out because it would contradict the fact that $s_{1}$ is maximal. Hence $\widetilde{\phi}^{\prime \star}\left(z_{\omega}\right) \neq 0^{\prime}$. This shows that $\phi_{1}^{\prime \star}(z)$ satisfies the definition of root function of order $s_{1}$, and hence it can replace $\phi_{1}^{\prime}(z)$ in (11).

While a canonical system of root functions (and also an extended canonical system of root functions) is not unique, the orders $s_{1} \geq s_{2} \geq \cdots \geq s_{m}>0=s_{m+1}=\cdots=s_{p}$ are uniquely determined by $C(z)$ at $z_{\omega}$, see Lemma 1.1 in Gohberg et al. (1993); they are called the partial multiplicities of $C(z)$ at $z_{\omega}$.

Finally, consider the local Smith factorization of $C(z)$ at $z=z_{\omega}$, see Gohberg et al. (1993), i.e., the factorization

$$
C(z)=E(z) M(z) H(z),
$$

where $M(z)=\operatorname{diag}\left(\left(z-z_{\omega}\right)^{s_{h}}\right)_{h=1, \ldots, p}$ is uniquely defined and contains the partial multiplicities $s_{1} \geq \cdots \geq s_{p}$ of $C(z)$ at $z=z_{\omega}$; the matrices $E(z), H(z)$ are analytic and invertible 
in a neighbourhood of $z=z_{\omega}$ and are non-unique. $M(z)$ is called the local Smith form of $C(z)$ at $z=z_{\omega} \cdot{ }^{6}$

Remark 22 (Extended canonical system of root functions in the I(1) VAR case). In the I(1) VAR case, see Example 1, the orders of an extended canonical system of root functions of $C(z)$ at 1 are $\left(s_{1}, \ldots, s_{r_{0}}, s_{r_{0}+1}, \ldots, s_{p}\right)=(1, \ldots, 1,0, \ldots, 0)$ and a possible choice of an extended canonical system of root functions corresponding to these unique orders is given by the $p$ rows in $\left(\beta_{0}, \beta_{1}\right)^{\prime}$.

Remark 23 (Extended canonical system of root functions in the I(2) VAR case). In the I(2) VAR case, see Example 2, the orders of an extended canonical system of root functions of $C(z)$ at 0 are $\left(s_{1}, \ldots, s_{r_{0}}, s_{r_{0}+1}, \ldots, s_{r_{0}+r_{1}}, s_{r_{0}+r_{1}+1}, \ldots, s_{p}\right)=(2, \ldots, 2,1, \ldots, 1,0, \ldots, 0)$ and a possible choice of an extended canonical system of root functions corresponding to these unique orders is given by the $p$ rows in $\left(\beta_{0}+(1-z)\left(\bar{\alpha}_{0}^{\prime} A_{1}\right)^{\prime}, \beta_{1}, \beta_{2}\right)^{\prime}$.

\section{Cointegrating Spaces}

Let $\phi(z)^{\prime}$ be a canonical system of root functions of $C(z)$ at $z_{\omega}$, see Definition 4 . Appendix A.2 shows that $\operatorname{row}_{G}\left(\phi(z)^{\prime}\right)$ with $G=F, F[z], F(z)$ are well defined sets of (generalized) root functions. This section argues that one could take any of them as a definition of 'cointegrating space' for multicointegrated systems. Note that

$$
\operatorname{row}_{F}\left(\phi(z)^{\prime}\right) \subset \operatorname{row}_{F[z]}\left(\phi(z)^{\prime}\right) \subset \operatorname{row}_{F(z)}\left(\phi(z)^{\prime}\right),
$$

so that the three definitions of cointegrating space are naturally nested. Remark that $\operatorname{row}_{F}\left(\phi(z)^{\prime}\right)$ is a vector space over $F, \operatorname{row}_{F[z]}\left(\phi(z)^{\prime}\right)$ is a free module over the ring $F[z]$ of polynomials in $z$ (which contains $\operatorname{row}_{F}\left(\phi(z)^{\prime}\right)$ ) and $\operatorname{row}_{F(z)}\left(\phi(z)^{\prime}\right)$ is a vector space over the field $F(z)$ of rationals functions of $z$ ((which contains $\operatorname{row}_{F[z]}\left(\phi(z)^{\prime}\right)$ and hence $\left.\operatorname{row}_{F}\left(\phi(z)^{\prime}\right)\right)$. Finally note the central role played by the canonical system of root functions $\phi(z)^{\prime}$ as a basis for these different spaces, which differ for the set of scalars chosen in linear combinations.

\subsection{The Cointegrating Space $\operatorname{row}_{F}\left(\phi(z)^{\prime}\right)$ as a Vector Space over $F$}

The cointegrating space $\operatorname{row}_{F}\left(\phi(z)^{\prime}\right)$, where $F=\mathbb{R}, \mathbb{C}$, is a vector space. In fact, the set of all $F$-linear combination of $\phi(z)^{\prime}$ produces a vector space, because $\operatorname{row}_{F}\left(\phi(z)^{\prime}\right)$ is closed under multiplication by a scalar in $F$ by Proposition A1 and with respect to vector addition, as a special case of Proposition A2.

In order to discuss the cointegrating spaces $\operatorname{row}_{F[z]}\left(\phi(z)^{\prime}\right)$ and $\operatorname{row}_{F(z)}\left(\phi(z)^{\prime}\right)$, the notion of generalized cointegrating vector is introduced, as the counterpart of the notion of generalized root function, see Definition A1.

Definition 5 (Generalized cointegrating vector at frequency $\omega$ ). Let $n \in \mathbb{Z}$ and $\zeta(z)^{\prime}$ be a cointegrating vector at frequency $\omega$ and order s, see Definition 2; then

$$
\xi(z)^{\prime}:=\left(1-z / z_{\omega}\right)^{n} \zeta(z)^{\prime}
$$

is called a generalized cointegrating vector at frequency $\omega$ with order s and exponent $n$.

\subsection{The Cointegrating Space $\operatorname{row}_{F[z]}\left(\phi(z)^{\prime}\right)$ as a Free Module over $F[z]$}

Consider next $\operatorname{row}_{F[z]}\left(\phi(z)^{\prime}\right) . F[z]$ is the polynomial ring formed as the set of polynomials in $z$ with coefficients in $F$. As it is well known, $F[z]$ is a ring but not a field (division ring), see e.g., Hungerford (1980), because polynomials, unlike rational functions, lack the multiplication inverse. The following propositions summarizes that $\operatorname{row}_{F[z]}\left(\phi(z)^{\prime}\right)$ is a free module over the ring $F[z]$ of polynomials.

Proposition $3\left(\operatorname{row}_{F[z]}\left(\phi(z)^{\prime}\right)\right.$ is a $F[z]$-module). Consider $\mathcal{G}=\operatorname{row}_{F[z]}\left(\phi(z)^{\prime}\right)$, where $\phi(z)^{\prime}$ is a canonical system of root functions of $C(z)$ at $z_{\omega}$ with coefficients in $F$, and where $F[z]$ is the 
ring of polynomials in $z$ with coefficients in $F$; then $\mathcal{G}$ is closed with respect to the vector sum, and it is closed under multiplication by a scalar polynomial in $F[z]$; hence $\mathcal{G}$ is a module over the ring $F[z]$ of polynomials.

Proof. By Propositions A1 and A2, $\mathcal{G}$ is closed under addition and under multiplication by a scalar polynomial in $F[z]$. One needs to verify that, see e.g., Definition IV.1.1 in Hungerford (1980), for $\zeta(z), \psi(z) \in \mathcal{G}$ and $1, a(z), b(z) \in F[z]$

$$
\begin{aligned}
a(z) \cdot\left(\zeta(z)^{\prime}+\psi(z)^{\prime}\right) & =a(z) \cdot \zeta(z)^{\prime}+a(z) \cdot \psi(z)^{\prime} \\
(a(z)+b(z)) \cdot \zeta(z)^{\prime} & =a(z) \cdot \zeta(z)^{\prime}+b(z) \cdot \zeta(z)^{\prime} \\
(a(z) b(z)) \cdot \zeta(z)^{\prime} & =a(z) \cdot\left(b(z) \cdot \zeta(z)^{\prime}\right) \\
1 \cdot \zeta(z)^{\prime} & =\zeta(z)^{\prime}
\end{aligned}
$$

where indicates multiplication by a scalar. The distributive properties in (13) are seen to be satisfied. This proves the statement.

\subsection{The Cointegrating Space $\operatorname{row}_{F(z)}\left(\phi(z)^{\prime}\right)$ as a Vector Space over $F(z)$}

Finally consider $\operatorname{row}_{F(z)}\left(\phi(z)^{\prime}\right)$. The set of scalars $F(z)$ is the field of rational functions in $z$ with coefficients in $F$. As it is well known, $F(z)$ is a field (division ring), see e.g., Hungerford (1980).

Remark 24 (Rational vectors without poles at $z_{\omega}$ ). Take $\zeta(L)^{\prime}$ to be a rational vector, i.e., of the form $\zeta(z)^{\prime}=\frac{1}{d(z)} b(z)^{\prime}$ where $d(z)$ is a monic polynomial and $b(z)^{\prime}$ is a $1 \times p$ vector polynomial, with $d(z)$ and $b(z)^{\prime}$ relatively prime, see Example A1. If $d(z)$ has no root equal to $z_{\omega}$, then $\zeta(z)^{\prime}$ is an analytic function on $D\left(z_{\omega}, \eta\right), \eta>0$, see Remark $A 1$ and Lemma A1; hence a special case of an analytic vector function $\zeta(z)^{\prime}$ is a rational vector with denominator $d(z)$ without roots equal to $z_{\omega}$.

Remark 25 (Rational vectors with poles at $z_{\omega}$ ). If $d(z)$ has one root equal to $z_{\omega}$ with multiplicity $m$, then $\zeta(z)^{\prime}$ has a pole of order $m$, and it is not an analytic function on some $D\left(z_{\omega}, \eta\right), \eta>0$; hence Definition 2 cannot be applied, because it requires $\zeta(z)^{\prime}$ to be analytic. However, one could remove the pole of order $m$ by defining $\xi(z)^{\prime}:=\left(1-z / z_{\omega}\right)^{m} \zeta(z)^{\prime}$, and use Definition 2 on $\xi(z)^{\prime}$, which is analytic function, as done in Definition 5.

Remark 26 (Representation for generic rational vectors). In the following, when dealing with rational vectors of the type $\zeta(z)^{\prime}=\frac{1}{d(z)} b(z)^{\prime}$, it is sufficient to consider the case where $d(z)$ does not have a root at $z_{\omega}$, thanks to Definition 5. In fact, let $d(z)$ be decomposed as $d(z)=$ $\left(1-z / z_{\omega}\right)^{m} d_{\star}(z)$ with $d_{\star}\left(z_{\omega}\right) \neq 0$ and $m \geq 0$; in this representation, $z_{\omega}$ is a root of $d(z)$ if and only if $m>0$ and it is not a root if and only if $m=0$. By Remark $24, \zeta(z)^{\prime}$ is a (generalized) cointegrating vector if and only if $\xi(z)^{\prime}:=\left(1-z / z_{\omega}\right)^{m} \zeta(z)^{\prime}=\frac{1}{d_{\star}(z)} b(z)^{\prime}$ is a cointegrating vector. Hence Definition 5 allows to concentrate on the case where the denominator has no root at $z_{\omega}$.

The following proposition summarizes that $\operatorname{row}_{F(z)}\left(\phi(z)^{\prime}\right)$ is a vector space over the field $F(z)$ of rational functions.

Proposition $4\left(\operatorname{row}_{F(z)}\left(\phi(z)^{\prime}\right)\right.$ is a vector space over $\left.F(z)\right)$. Let $\mathcal{H}=\operatorname{row}_{F(z)}\left(\phi(z)^{\prime}\right)$ where $\phi(z)^{\prime}$ is a canonical system of root functions of $C(z)$ at $z_{\omega}$ with coefficients in $F$, where $F(z)$ is the field of rational function in $z$ with coefficients in $F$; then $\mathcal{H}$ is closed with respect to the vector sum, and under multiplication by a scalar rational function in $F(z)$, and $\mathcal{H}$ is a vectors space over the field $F(z)$ of rational functions.

Proof. $\mathcal{H}$ is closed with respect to multiplication by a rational function in $F(z)$, see Proposition A1, and with respect to vector addition, see Proposition A2. One can ver- 
ify for $\zeta(z), \psi(z) \in \mathcal{H}$ and $1, a(z), b(z) \in F(z)$, that the distribution equalities in (13) are satisfied. Because $F(z)$ is a field, then $\mathcal{H}$ is a vector space over $F(z)$.

\section{The Local Rank Factorization}

This section shows how to explicitly obtain a canonical system of root functions $\phi(z)^{\prime}$ or an extended canonical system of root functions $\left(\phi(z), a_{\perp}\right)^{\prime}$ for a generic VAR process

$$
A(L) x_{t}=\varepsilon_{t}, \quad A_{0} \neq 0, \quad \operatorname{det} A_{0}=0,
$$

with $A(z)$ analytic for all $z \in D(0,1+\eta), \eta>0$, having roots at $z=z_{\omega}=e^{i \omega}$ and at $z$ with $|z|>1$, see Remarks 1 and 2 .

The derivation of the Granger representation theorem involves the inversion of the matrix function

$$
A(z)=\sum_{n=0}^{\infty}\left(z-z_{\omega}\right)^{n} A_{n}, \quad A_{n} \in \mathbb{C}^{p \times p}, \quad A_{0} \neq 0, \quad \operatorname{det} A_{0}=0,
$$

in $D\left(z_{\omega}, \eta\right)$. This includes the case of matrix polynomials $A(z)$, in which the degree of $A(z)$ is finite, $k$ say, with $A_{n}=0$ for $n>k .^{7}$

The inversion of $A(z)$ around the singular point $z=z_{\omega}$ yields an inverse with a pole of some order $d=1,2, \ldots$ at $z=z_{\omega}$; an explicit condition on the coefficients $\left\{A_{n}\right\}_{n=0}^{\infty}$ in (15) for $A(z)^{-1}$ to have a pole of given order $d$ is described in Theorem 2 below; this is indicated as the $\operatorname{POLE}(d)$ condition in the following. Under the $\operatorname{POLE}(d)$ condition, $A(z)^{-1}$ has Laurent expansion around $z=z_{\omega}$ given by

$$
A(z)^{-1}=:\left(z-z_{\omega}\right)^{-d} C(z)=\sum_{n=0}^{\infty}\left(z-z_{\omega}\right)^{n-d} C_{n}, \quad C_{0} \neq 0, \quad \operatorname{det} C_{0}=0
$$

Note that $C\left(z_{\omega}\right)=C_{0} \neq 0$ and $C(z)$ is expanded around $z=z_{\omega}$. In the following, the coefficients $\left\{C_{n}\right\}_{n=0}^{\infty}$ are called the Laurent coefficients. The first $d$ of them, $\left\{C_{n}\right\}_{n=0}^{d-1}$, make up the principal part and characterize the singularity of $A(z)^{-1}$ at $z=z_{\omega}$.

The following result is taken from Franchi and Paruolo (2019) Theorem 3.3. ${ }^{8}$

Theorem $2\left(\operatorname{POLE}(d)\right.$ condition). Consider $A(z)$ defined in (15); let $0<r_{0}:=\operatorname{rank} A_{0}<p$, $r_{0}^{\max }:=p$ and define $\alpha_{0}, \beta_{0}$ by the rank factorization $A_{0}=-\alpha_{0} \beta_{0}^{\prime}$. Moreover, for $j=1,2, \ldots$ define $\alpha_{j}, \beta_{j}$ by the rank factorization

$$
P_{a_{j \perp}} A_{j, 1} P_{b_{j \perp}}=-\alpha_{j} \beta_{j}^{\prime}, \quad a_{j}:=\left(\alpha_{0}, \ldots, \alpha_{j-1}\right), \quad b_{j}:=\left(\beta_{0}, \ldots, \beta_{j-1}\right),
$$

where $P_{x}$ denotes the orthogonal projection onto the space spanned by the columns of $x$ and

$$
A_{h+1, n}:=\left\{\begin{array}{cl}
A_{n} & \text { for } h=0 \\
A_{h, n+1}+A_{h, 1} \sum_{i=0}^{h-1} \bar{\beta}_{i} \bar{\alpha}_{i}^{\prime} A_{i+1, n} & \text { for } h=1,2, \ldots
\end{array}, \quad n=0,1, \ldots\right.
$$

Finally, let

$$
r_{j}:=\operatorname{rank}\left(P_{a_{j \perp}} A_{j, 1} P_{b_{j \perp}}\right), \quad r_{j}^{\max }:=p-\sum_{i=0}^{j-1} r_{i} .
$$

Then, a necessary and sufficient condition for $A(z)$ to have an inverse with pole of order $d=1,2, \ldots$ at $z=z_{\omega}$-called POLE $(d)$ condition - is that

$$
\left\{\begin{array}{ll}
r_{j}<r_{j}^{\max } & (\text { reduced rank condition }) \text { for } j=0, \ldots, d-1 \\
r_{d}=r_{d}^{\max } & \text { (full rank condition }) \text { for } j=d
\end{array} .\right.
$$


Observe that because rank $P_{a_{j \perp}} A_{j, 1} P_{b_{j \perp}}=\operatorname{rank} a_{j \perp}^{\prime} A_{j, 1} b_{j \perp}$, one has $r_{j}=\operatorname{rank} a_{j \perp}^{\prime} A_{j, 1} b_{j \perp} ;$ hence $d=1$ if and only if

$$
r_{1}=r_{1}^{\max }, \quad \text { where } r_{1}=\operatorname{rank} \alpha_{0 \perp}^{\prime} A_{1} \beta_{0 \perp} \quad \text { and } \quad r_{1}^{\max }=p-r_{0} .
$$

This corresponds to the condition in Howlett (1982, Theorem 3) and to the $I(1)$ condition in Johansen (1991, Theorem 4.1). Similarly, one has $d=2$ if and only if $r_{1}<r_{1}^{\max }$,

$$
r_{2}=r_{2}^{\max }, \quad \text { where } r_{2}=\operatorname{rank} a_{2 \perp}^{\prime}\left(A_{2}+A_{1} \bar{\beta}_{0} \bar{\alpha}_{0}^{\prime} A_{1}\right) b_{2 \perp} \quad \text { and } \quad r_{2}^{\max }=p-r_{0}-r_{1},
$$

which corresponds to the $I(2)$ condition in Johansen (1992, Theorem 3).

Theorem 2 is thus a generalization of the Johansen's $I(1)$ and $I(2)$ conditions and shows that, in order to have a pole of order $d$ in the inverse, one needs $d+1$ rank conditions on $A(z)$ : The first $j=0, \ldots, d-1$ are reduced rank conditions, $r_{j}<r_{j}^{\max }$, that establish that the order of the pole is greater than $j$; the last one is a full rank condition, $r_{d}=r_{d}^{\max }$, that establishes that the order of the pole is exactly equal to $d$. These requirements make up the POLE $(d)$ condition.

The following result is also taken from Franchi and Paruolo (2019). ${ }^{9}$

Theorem 3 (Local Smith factorization). Consider $A(z)$ and the other related quantities defined in Theorem 16; for $j=0, \ldots, d$, define the $r_{j} \times p$ matrix functions $\gamma_{j}(z)^{\prime}$ as follows

$$
\gamma_{j, 0}^{\prime}:=\beta_{j,}^{\prime}, \quad \gamma_{j, n}^{\prime}:=-\bar{\alpha}_{j}^{\prime} A_{j+1, n}, \quad n=1,2, \ldots \quad \gamma_{j}(z)^{\prime}:=\sum_{n=0}^{\infty}\left(z-z_{\omega}\right)^{n} \gamma_{j, n}^{\prime},
$$

and define the $p \times p$ matrix functions $\Gamma(z)$ and $\Lambda(z)$ as follows

$$
\Gamma(z):=\left(\begin{array}{c}
\gamma_{0}(z)^{\prime} \\
\vdots \\
\gamma_{d}(z)^{\prime}
\end{array}\right), \quad \Lambda(z):=\left(\begin{array}{ccc}
\left(z-z_{\omega}\right)^{0} I_{r_{0}} & & \\
& \ddots & \\
& & \left(z-z_{\omega}\right)^{d} I_{r_{d}}
\end{array}\right) .
$$

Then $\Gamma(z), \Xi(z):=A(z) \Gamma(z)^{-1} \Lambda(z)^{-1}$ are analytic and invertible on $D\left(z_{\omega}, \eta\right), \eta>0$, and $\Lambda(z)$ is the local Smith form of $A(z)$ at $z_{\omega}, A(z)=\Xi(z) \Lambda(z) \Gamma(z)$. Moreover one can choose the factors $E(z), M(z), H(z)$ for the local Smith factorization of $C(z)$ defined in (16), see (12), as

$$
E(z)=\Gamma(z)^{-1}, \quad M(z)=\left(z-z_{\omega}\right)^{d} \Lambda(z)^{-1}, \quad H(z)=\Xi(z)^{-1} .
$$

Theorem 3 shows that the LRF fully characterizes the elements of the local Smith factorization of $C(z)$ at $z_{\omega}$. In fact, the values of $j$ with $r_{j}>0$ in the LRF provide the distinct partial multiplicities of $C(z)$ at $z_{\omega}$ and $r_{j}$ gives the number of partial multiplicities that are equal to a given $j$; this characterizes the local Smith form $\Lambda(z)$. Moreover, it also provides the constructions of an extended canonical system of root functions.

Remark that the $j$-th block of rows in $\Gamma(z) C(z)=\left(z-z_{\omega}\right)^{d} \Lambda(z)^{-1} \Xi(z)^{-1}$ can be written as

$$
\gamma_{j}(z)^{\prime} C(z)=\left(z-z_{\omega}\right)^{d-j} \widetilde{\gamma}_{j}(z)^{\prime}, \quad j=0, \ldots, d,
$$

where $\gamma_{j}\left(z_{\omega}\right)^{\prime}=\beta_{j}^{\prime}$ and $\widetilde{\gamma}_{j}\left(z_{\omega}\right)^{\prime}$ have full row rank; here $\widetilde{\gamma}_{j}(z)^{\prime}$ denotes the corresponding block of rows in $\Xi(z)^{-1}$. This shows that $\gamma_{j}(z)^{\prime}$ are $r_{j}$ root functions of order $d-j$ of $C(z)$.

The next result presents the Triangular representation as proved in Franchi and Paruolo (2019, Corollary 4.6). 
Proposition 5 (Triangular representation). Let $x_{t}$ in (14) satisfy the POLE $(d)$ condition on $A(z)$ and define

$$
\begin{aligned}
\Gamma_{\circ}(L): & =\left(\begin{array}{c}
\phi(L)^{\prime} \\
-\beta_{d}^{\prime}--
\end{array}\right), \\
\phi(L)^{\prime} & :=\left(\begin{array}{c}
\gamma_{0}^{(d-1)}(L)^{\prime} \\
\gamma_{1}^{(d-2)}(L)^{\prime} \\
\vdots \\
\gamma_{d-1}^{(0)}(L)^{\prime}
\end{array}\right)=\left(\begin{array}{c}
\beta_{0}^{\prime}+\sum_{k=1}^{d-1}\left(-z_{\omega}\right)^{k} \gamma_{0, k}^{\prime} \Delta_{\omega}^{k} \\
\beta_{1}^{\prime}+\sum_{k=1}^{d-2}\left(-z_{\omega}\right)^{k} \gamma_{1, k}^{\prime} \Delta_{\omega}^{k} \\
\vdots \\
\beta_{d-1}^{\prime}
\end{array}\right),
\end{aligned}
$$

where $\gamma_{j}^{(d-j-1)}(z)^{\prime}=\sum_{k=0}^{d-j-1} \gamma_{j, k}^{\prime}\left(z-z_{\omega}\right)^{k}$ is the truncation of order $d-j-1$ of the root functions $\gamma_{j}(z)^{\prime}$ in (20). Then $x_{t}$ is $I(d)$ and it admits the Triangular Representation

$$
\Lambda(L) \Gamma_{\circ}(L) x_{t} \sim I(0)
$$

where no linear combination exists of the l.h.s. that is integrated of lower order.

Observe that the canonical system of root functions $\phi(z)^{\prime}$ in (23) is not unique and not of minimal polynomial order, as discussed in the next section. The following example applies the above concepts in the I(2) VAR case.

Example 3 (I(2) VAR example continued). Consider Example 2. Applying truncation to the rows of $\left(\beta_{0}^{\prime}+\Delta \bar{\alpha}_{0}^{\prime} A_{1}\right)$, see Propositions 5 and $A 3$, one finds that the columns in $\beta_{0}^{\prime}$ are root functions of $C(z)$ at $\omega=0$ of order at least $\min (2,1)=1$. Consider now one row in $\left(\beta_{0}^{\prime}+\Delta \bar{\alpha}_{0}^{\prime} A_{1}+\Delta^{2} A^{\prime}\right)$ for some matrix $A$; this root function is of order 2 by Remark 23 , and its truncation to degree 1, i.e., to the corresponding row of $\left(\beta_{0}^{\prime}+\Delta \bar{\alpha}_{0}^{\prime} A_{1}\right)$ is still of order 2 by Propositions 5 and $A 3$, Finally consider one row in $\left(\beta_{0}^{\prime}+\Delta A^{\prime}\right)$, which gives a root function of order at least 1 ; its truncation to a polynomial of degree 0 gives the corresponding row of $\beta_{0}^{\prime}$, which has order at least 1 by Propositions 5 and A3. In fact the rows of $\beta_{0}^{\prime}$ give root functions of order equal to 1 or to 2, when the corresponding entries in $\bar{\alpha}_{0}^{\prime} A_{1}$ in $\left(\beta_{0}^{\prime}+\Delta \bar{\alpha}_{0}^{\prime} A_{1}\right)$ are equal to 0 , as discussed below.

\section{Minimal Bases}

This section describes the algorithm of Forney (1975) to reduce the basis $\phi(z)^{\prime}$ to minimal order, using the generic notation of $b(z)^{\prime}$ in place of $\phi(z)^{\prime}$. The generic basis $b(z)^{\prime}$ is assumed to be rational and of dimension $r \times p$. This algorithm exploits the nesting $\operatorname{row}_{F}\left(b(z)^{\prime}\right) \subset \operatorname{row}_{F[z]}\left(b(z)^{\prime}\right) \subset \operatorname{row}_{F(z)}\left(b(z)^{\prime}\right)$. In the following, the $j$-row of $b(z)^{\prime}$ is indicated as $b_{j}(z)^{\prime}$, which is the $j$-th element of the basis, $j=1, \ldots, r$. Various modifications of the original basis $b^{(0)}(z)^{\prime}:=b(z)^{\prime}$ are indicated as $b^{(h)}(z)^{\prime}$ for $h=1,2,3$.

Definition 6 (Degree of $\left.b(z)^{\prime}\right)$. If $b(z)^{\prime}$ is a polynomial basis, the degree $v_{j}$ of its $j$-th row, indicated as $v_{j}:=\operatorname{deg} b_{j}(z)^{\prime}$, is defined as the maximum degree of its elements, and the degree $v$ of $b(z)^{\prime}$ is defined as $v:=\operatorname{deg} b(z)^{\prime}:=\sum_{j=1}^{r} v_{j}$, i.e., the sum of the degrees of its rows.

The reduction algorithm proposed by Forney (1975, pp. 497-98) consists of the following 3 steps.

Step 1 If $b^{(0)}(z)^{\prime}$ is not polynomial, multiply each row by the least common denominator of each row to obtain a polynomial basis $b^{(1)}(z)^{\prime}$.

Step 2 Reduce row orders in $b^{(1)}(z)^{\prime}$ by taking $F[z]$-linear combinations.

Step 3 Reduce $b^{(2)}(z)^{\prime}$ to a basis $b^{(3)}(z)^{\prime}$ with a full-row-rank high order coefficient matrix, i.e., a "row proper" basis.

This procedure gives a final basis $b^{(3)}(z)^{\prime}$ which has lowest degree, see Forney (1975) Section 3. 
Remark 27 (Spaces and algorithm). Step 1 works on $\operatorname{row}_{F(z)}\left(\phi(z)^{\prime}\right)$, Step 2 works on $\operatorname{row}_{F[z]}\left(\phi(z)^{\prime}\right)$, Step 3 uses F-linear combinations on $Q(z) \phi(z)^{\prime}$ with appropriate square polynomial matrices $Q(z)$.

\subsection{Step 1}

If $b^{(0)}(z)^{\prime}$ is polynomial, the algorithm sets $b^{(1)}(z)^{\prime}=b^{(0)}(z)^{\prime}$; otherwise $b^{(0)}(z)^{\prime}$ is rational, and its $j$-th row $b_{j}(z)^{\prime}$ has representation $b_{j}(z)^{\prime}=\frac{1}{a_{j}(z)} c_{j}(z)^{\prime}$, where $c_{j}(z)^{\prime}$ is a polynomial row vector and $a_{j}(z)$ is a scalar polynomial, and $c_{j}(z)$ and $a_{j}(z)$ are relatively prime. The first step consist in computing $b^{(1)}(z)^{\prime}=\operatorname{diag}\left(a_{1}(z), \ldots, a_{r}(z)\right) b^{(0)}(z)^{\prime}$, where $Q(z):=\operatorname{diag}\left(a_{1}(z), \ldots, a_{r}(z)\right)$ is a square polynomial matrix of dimension $r$.

\subsection{Step 2}

The second step reduces the degree of the rows in $b^{(1)}(z)^{\prime}$. This involves finding specific points $z_{h}, h=1, \ldots, k$, at which $\operatorname{rank}\left(b^{(1)}\left(z_{h}\right)^{\prime}\right)<r$. To find them, one can calculate the greatest common divisor $\ell(z)$ of all $r \times r$ minors of $b^{(1)}(z)^{\prime}$. If $\ell(z)=1$ this step is complete, and the algorithm sets $b^{(2)}(z)^{\prime}=b^{(1)}(z)^{\prime}$; otherwise one computes the zeros of $\ell(z), z_{1}, \ldots, z_{k}$ say, where $z_{h} \in \mathbb{C}, h=1, \ldots, k$. The following substep is then applied to each root $z_{h}$ sequentially, $h=1, \ldots, k$.

Denote by $w(z)^{\prime}$ the current basis; this will be replaced by $\kappa(z)^{\prime}$ at the end of this substep. For $h=1$, one has $w(z)^{\prime}=b^{(1)}(z)^{\prime}$. For $z=z_{h}$, all minors of order $r$ of $w\left(z_{h}\right)^{\prime}$ vanish, which means that $w\left(z_{h}\right)^{\prime}$ is singular, i.e., it has reduced rank and rank factorization $w\left(z_{h}\right)^{\prime}=\psi a^{\prime}$, say, where $\psi, a$ are full column rank. Let $c^{\prime}:=\left(c_{1}, \ldots, c_{p}\right)$ be one row in $\psi_{\perp}^{\prime}$. Indicate by $A_{c}:=\left\{i: c_{i} \neq 0\right\}$ the set of its non-zero coefficients, and let $v_{i_{0}}:=\max _{i \in A_{c}}\left\{v_{i}\right\}$ be the maximal degree of rows in $w(z)^{\prime}$ with nonzero coefficient in $c^{\prime}$.

This substep consists of replacing row $i_{0}$ of $w(z)^{\prime}$ with $c^{\prime} w(z)^{\prime} /\left(z-z_{h}\right)$, which is still a polynomial vector. In fact $c^{\prime} w\left(z_{h}\right)^{\prime}=0^{\prime}$, so that $c^{\prime} w\left(z_{h}\right)^{\prime}$ has representation $c^{\prime} w\left(z_{h}\right)^{\prime}=$ $\left(z-z_{h}\right) \tau(z)^{\prime}$ with $\tau(z)^{\prime}$ a polynomial vector, so that $c^{\prime} w(z)^{\prime} /\left(z-z_{h}\right)=\tau(z)^{\prime}$. This defines $\kappa(z)^{\prime}$ in terms of $w(z)^{\prime}$ as $\kappa(z)^{\prime}=B(z)^{-1} Q w(z)^{\prime}$ where $Q$ is an $r \times r$ square matrix, equal to $I_{r}$ except for row $i_{0}$, equal to $c^{\prime}$, and where $B(z)$ is a diagonal matrix equal to $I_{r}$ except for having $z-z_{h}$ in its $i_{0}$-th position on the diagonal. Note that $Q$ is nonsingular, because $c_{i_{0}} \neq 0$. The same procedure is applied to each row $c^{\prime}$ of $\psi_{\perp}^{\prime}$.

This substep is repeated for all $z_{j}, j=1, \ldots, k$. The condition on the minors in then recalculated and the substep repeated for the new roots, until the greatest common divisor $\ell(z)$ of all $r \times r$ minors of $\kappa(z)^{\prime}$ is 1 . When this is the case, Step 2 sets $b^{(2)}(z)^{\prime}=\kappa(z)^{\prime}$.

\subsection{Step 3}

The last step operates on the high order coefficient matrix, repeating the following substep. Let $w(z)^{\prime}$ indicate $b^{(2)}(z)^{\prime}$ at the beginning of the substep, which will be replaced by $\kappa(z)^{\prime}$ at the end of it. Let $v_{i}$ be the order of the $i$-th row of $w(z)^{\prime}$, indicated as $w_{i}(z)^{\prime}=\sum_{j=0}^{v_{i}}\left(z-z_{\omega}\right)^{j} w_{i j}^{\prime}$. The high-order matrix is defined as the $r \times p$ matrix $w_{*}^{\prime}:=\left(w_{1 v_{1}}, \ldots, w_{r v_{r}}\right)^{\prime}$ composed of the coefficient matrix of the highest degree of $\left(z-z_{\omega}\right)$ for each row of $w(z)^{\prime}$.

A necessary and sufficient condition for $w_{*}^{\prime}$ to be of full rank is that the order of $w(z)^{\prime}$ is equal to the maximum order of its $r \times r$ minors. If this is not the case, $w_{*}^{\prime}$ is singular, i.e., it has rank factorization $w_{*}^{\prime}=\psi a^{\prime}$ with $\psi$ and $a$ of full column rank. Hence one can choose a vector $c^{\prime}:=\left(c_{1}, \ldots, c_{p}\right)$ as one row in $\psi_{\perp}^{\prime}$ for which one has $c^{\prime} w_{*}^{\prime}=0^{\prime}$. 
As before, let $A_{c}:=\left\{i: c_{i} \neq 0\right\}$ and define $v_{i_{0}}:=\max _{i \in A_{c}}\left\{v_{i}\right\}$. Let also $n_{i}:=v_{i_{0}}-v_{i}$, note that $n_{i} \geq 0$ for $i \in A_{c}$ and let $Q(z):=\operatorname{diag}\left(\left(z-z_{\omega}\right)^{n_{1}}, \ldots\left(z-z_{\omega}\right)^{n_{r}}\right)$. Row $i_{0}$ in $w(z)^{\prime}$ is replaced by

$$
\begin{aligned}
q(z)^{\prime}:=c^{\prime} Q(z) w(z)^{\prime} & =\sum_{i \in A_{c}} c_{i} \sum_{j=0}^{v_{i}}\left(z-z_{\omega}\right)^{j+n_{i}} w_{i, j}^{\prime}=\sum_{i \in A_{c}} c_{i} \sum_{s=n_{i}}^{v_{i_{0}}}\left(z-z_{\omega}\right)^{s} w_{i, s-n_{i}}^{\prime} \\
& =\sum_{j=0}^{v_{i_{0}}-1}\left(z-z_{\omega}\right)^{j} q_{j}^{\prime}+\left(z-z_{\omega}\right)^{v_{i_{0}}} c^{\prime} w_{*}^{\prime}=\sum_{j=0}^{v_{i_{0}}-1}\left(z-z_{\omega}\right)^{j} q_{j}^{\prime}
\end{aligned}
$$

where $s$ in the last expression in the first line is defined as $j+n_{i}$ and $q_{j}^{\prime}:=\sum_{i \in A_{c}} c_{i} w_{i, n_{i}+j}^{\prime}$.

The central expression in (24) shows that $q(z)^{\prime}$ is polynomial because $n_{i} \geq 0$ in the exponents of $\left(z-z_{\omega}\right)$. In order to see that the degree of $q(z)^{\prime}$ is also lower than $v_{i_{0}}$, one can note that the the high order coefficient in (25), which correspond to $s=v_{i_{0}}$ in (24), equals $\sum_{i \in A_{c}} c_{i} w_{i, v_{i}}^{\prime}=c^{\prime} w_{*}^{\prime}=0^{\prime}$. This implies that the order of $q(z)^{\prime}$ is lower than $v_{i_{0}}$, and that replacing row $i_{0}$ of $w(z)^{\prime}$ with $q(z)^{\prime}$ reduces the order of the vector.

This defines $\kappa(z)^{\prime}$ in terms of $w(z)^{\prime}$ as $\kappa(z)^{\prime}=N Q(z) w(z)^{\prime}$ where $N$ is an $r \times r$ square matrix, equal to $I_{r}$ except for row $i_{0}$, equal to $c^{\prime}$. Note that $N$ is nonsingular, because $c_{i_{0}} \neq 0$. This process is repeated for all the rows $c^{\prime}$ in $\psi_{\perp}^{\prime}$. Next set $w(z)^{\prime}=\kappa(z)^{\prime}$ and repeat until the high order coefficient matrix has full rank. When this is the case, Step 3 sets $b^{(3)}(z)^{\prime}=\kappa(z)^{\prime}$.

\section{From a Canonical System of Root Functions to a Minimal Basis for I(2) VAR}

This section applies the algorithm of Forney reviewed in Section 7 to $\phi(z)^{\prime}$ in (23) to reduce the basis to minimal order in the $I(2)$ VAR example at frequency $\omega=0$. This application leads to the separation of the cases of

(i) non-polynomial cointegrating relations reducing the order of integration from 2 to 0 ;

(ii) polynomial cointegrating relations reducing the order of integration from 2 to 0.

The process of obtaining minimal bases does not lead to a unique choice of basis; this leaves open the choice of how to further restrict the basis to obtain uniqueness. Forney (1975) obtains uniqueness requiring the minimal basis to be in upper echelon form. Other sets of restrictions can also be considered. For the sake of brevity, the restrictions on how to obtain a unique minimum basis are not further discussed here.

\subsection{Step 1 in I(2) VAR}

Consider the triangular representation of an $I(2)$ system, see (23):

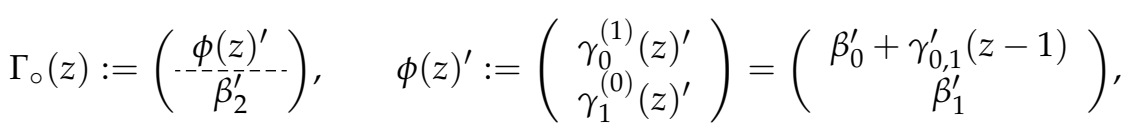

and apply the algorithm of Forney (1975) to $b^{(0)}(z)^{\prime}:=\phi(z)^{\prime}$. Because $b^{(0)}(z)^{\prime}$ is already polynomial, one has $b^{(1)}(z)^{\prime}=b^{(0)}(z)^{\prime}=\phi(z)^{\prime}$.

\subsection{Step 2 in I(2) VAR}

Next consider Step 2, and set $w(z)^{\prime}=b^{(1)}(z)^{\prime}$. One wishes to find some zero $z_{h}$ and some corresponding $c^{\prime}$ so as have $c^{\prime} w\left(z_{h}\right)^{\prime}=0^{\prime}$. Denoting $u=z_{h}-1$, one hence needs to find the pair $\left(u, c^{\prime}\right)$ such that

$$
c^{\prime}\left(\left(\begin{array}{l}
\beta_{0}^{\prime} \\
\beta_{1}^{\prime}
\end{array}\right)+\left(\begin{array}{c}
\gamma_{0,1}^{\prime} \\
0
\end{array}\right) u\right)=0,
$$


where $u$ is a scalar. Note that $u=0$ is not a possible zero of (27), because $w\left(z_{\omega}\right)=\left(\beta_{0}, \beta_{1}\right)^{\prime}$ is of full row rank, so that $u \neq 0$. Post-multiplying (27) by the square non-singular matrix $\left(\bar{\beta}_{0}, \bar{\beta}_{1}, \bar{\beta}_{2}\right)$ one finds

$$
c^{\prime}\left(\left(\begin{array}{ccc}
I_{r_{0}} & 0 & 0 \\
0 & I_{r_{1}} & 0
\end{array}\right)+\left(\begin{array}{ccc}
\gamma_{0,1}^{\prime} \bar{\beta}_{0} & \gamma_{0,1}^{\prime} \bar{\beta}_{1} & \gamma_{0,1}^{\prime} \bar{\beta}_{2} \\
0 & 0 & 0
\end{array}\right) u\right)=0 .
$$

Hence, partitioning $c^{\prime}$ as $c^{\prime}=\left(\varsigma^{\prime}, \theta^{\prime}\right)$ where $\varsigma^{\prime}$ is $1 \times r_{0}$, one finds that the second set of equations gives $\theta^{\prime}=0^{\prime}$ and the first one, substituting the expression of $\gamma_{0,1}^{\prime}=-\bar{\alpha}_{0}^{\prime} A_{1}$ given in Theorem 3, implies

$$
\begin{aligned}
\varsigma^{\prime} \bar{\alpha}_{0}^{\prime} A_{1} \bar{\beta}_{0} & =\lambda \varsigma^{\prime}, \quad \lambda:=u^{-1} \neq 0, \\
\varsigma^{\prime} \bar{\alpha}_{0}^{\prime} A_{1}\left(\bar{\beta}_{1}, \bar{\beta}_{2}\right) & =0,
\end{aligned}
$$

where $\lambda=u^{-1} \neq 0$ in (29); note also that $u \neq 0$ has been simplified in (30). This proves the following proposition.

Proposition 6 (Step 2 condition in I(2)). A necessary and sufficient condition for Step 2 to be non-empty is that (29), (30) hold simultaneously, i.e., that $\left(\lambda, \varsigma^{\prime}\right)$ is a non-zero eigenvalue-left eigenvector pair of $\bar{\alpha}_{0}^{\prime} A_{1} \bar{\beta}_{0}$, and the left eigenvector $v^{\prime}$ is orthogonal to $\bar{\alpha}_{0}^{\prime} A_{1}\left(\bar{\beta}_{1}, \bar{\beta}_{2}\right)$. If this is the case, for each pair $\left(\lambda, \varsigma^{\prime}\right)$ one has

$$
\varsigma^{\prime} \bar{\alpha}_{0}^{\prime} A_{1}=\varsigma^{\prime} \bar{\alpha}_{0}^{\prime} A_{1} P_{\beta_{0}}=\lambda \varsigma^{\prime} \beta_{0}^{\prime} .
$$

Observe that from (27), using $c^{\prime}=\left(\varsigma^{\prime}, \theta^{\prime}\right)$ and $z-1=z-z_{h}+u$ with $u=z_{h}-1$, one finds

$$
\begin{aligned}
c^{\prime} w(z)^{\prime} & =\varsigma^{\prime} \beta_{0}^{\prime}-\left(z-z_{h}+u\right) \varsigma^{\prime} \bar{\alpha}_{0}^{\prime} A_{1} \\
& =\varsigma^{\prime}\left(\beta_{0}^{\prime}-\bar{\alpha}_{0}^{\prime} A_{1} u\right)-\left(z-z_{h}\right) \varsigma^{\prime} \bar{\alpha}_{0}^{\prime} A_{1}=-\left(z-z_{h}\right) \varsigma^{\prime} \bar{\alpha}_{0}^{\prime} A_{1},
\end{aligned}
$$

where the last equality follows from (31). This shows that under the necessary and sufficient condition in Proposition 6, there is a linear combination $c^{\prime}$ of $w^{\prime}(z)$ where one can factor $z-z_{h}$ out of $c^{\prime} w^{\prime}(z)$, which reduces the order from 1 to 0 . Here $c^{\prime} w^{\prime}(z)$, which has degree equal to 1 , is replaced by $c^{\prime} w^{\prime}(z) /\left(z-z_{h}\right)=-\varsigma^{\prime} \bar{\alpha}_{0}^{\prime} A_{1}=-\lambda \varsigma^{\prime} \beta_{0}^{\prime}$, which has degree 0 . Note that from (31) the new cointegrating relation is in the span of $\beta_{0}^{\prime}$.

This can be done for all pairs $\left(\lambda, \varsigma^{\prime}\right)$. Let $\left(\lambda, \varsigma_{j}^{\prime}\right)$ be all the pairs $\left(\lambda, \varsigma^{\prime}\right)$ satisfying the assumptions of Proposition $6, j=1, \ldots, s$, and let $q^{\prime}:=\left(\lambda_{1} \varsigma_{1}, \ldots, \lambda_{k} \varsigma_{s}\right)^{\prime}$. Choose also $a^{\prime}$ as some matrix $(r-s) \times r$ matrix such that $(q, a)$ is square and nonsingular; many matrices satisfy this criterion, including $q_{\perp}$. The output of Step 2 can be expressed as the following choice of $b^{(2)}(z)^{\prime}$ :

$$
b^{(2)}(z)^{\prime}=\left(\begin{array}{c}
a^{\prime} \beta_{0}^{\prime}-(z-1) a^{\prime} \bar{\alpha}_{0}^{\prime} A_{1} \\
q^{\prime} \beta_{0}^{\prime} \\
\beta_{1}^{\prime}
\end{array}\right) .
$$

Remark $28\left(\mathrm{CI}(2,2)\right.$ cointegration). This step brings out from $\phi(z)^{\prime}$ some cointegrating relations $q^{\prime} \beta_{0}^{\prime}$ that map the I(2) variables directly to I(0) without the help of first differences $\Delta$.

\subsection{Step 3 in I(2) VAR}

Consider $b^{(2)}(z)^{\prime}$ in (33) and its high order coefficient matrix

$$
w_{*}^{\prime}=\left(\begin{array}{c}
-a^{\prime} \bar{\alpha}_{0}^{\prime} A_{1} \\
q^{\prime} \beta_{0}^{\prime} \\
\beta_{1}^{\prime}
\end{array}\right) .
$$


Step 3 requires to find a nonzero matrix $c^{\prime}$ such that $c^{\prime} w_{*}^{\prime}=0^{\prime}$. Recall that $\left(\bar{\beta}_{0}, \bar{\beta}_{1}, \bar{\beta}_{2}\right)$ is square and nonsingular; hence $c^{\prime} w_{*}^{\prime}=0^{\prime}$ if and only if, partitioning $c^{\prime}$ as $c^{\prime}=\left(\zeta^{\prime}, \rho^{\prime}, \tau^{\prime}\right)$ one has

$$
0^{\prime}=c^{\prime} w_{*}^{\prime}\left(\bar{\beta}_{0}, \bar{\beta}_{1}, \bar{\beta}_{2}\right)=\left(\zeta^{\prime}, \rho^{\prime}, \tau^{\prime}\right)\left(\begin{array}{ccc}
-a^{\prime} \bar{\alpha}_{0}^{\prime} A_{1} \bar{\beta}_{0} & -a^{\prime} \bar{\alpha}_{0}^{\prime} A_{1} \bar{\beta}_{1} & -a^{\prime} \bar{\alpha}_{0}^{\prime} A_{1} \bar{\beta}_{2} \\
q^{\prime} & 0 & 0 \\
0 & I_{r_{1}} & 0
\end{array}\right) .
$$

This equality can be written as

$$
\begin{aligned}
\zeta^{\prime} a^{\prime} \bar{\alpha}_{0}^{\prime} A_{1}\left(\bar{\beta}_{0}, \bar{\beta}_{1}\right) & =\left(\rho^{\prime} q^{\prime}, \tau^{\prime}\right), \\
\zeta^{\prime} a^{\prime} \bar{\alpha}_{0}^{\prime} A_{1} \bar{\beta}_{2} & =0 .
\end{aligned}
$$

Remark 29 (Further degree reductions). Equation (35) requires $\zeta^{\prime}$ to be orthogonal to remaining part of the multicointegrating coefficient $a^{\prime} \bar{\alpha}_{0}^{\prime} A_{1}$ in direction of $\beta_{2}$. In addition $\zeta^{\prime}$ also needs to satisfy (34). For some configurations of dimensions, (34) could be solvable for $\left(\rho^{\prime}, \tau^{\prime}\right)$ in terms of other quantities; in this case (34) would not impose further restrictions.

Let also $\vartheta^{\prime}$ be any complementary matrix such that $(\zeta, \vartheta)$ is square and nonsingular; one possible choice of $\vartheta$ is $\zeta_{\perp}$. The output of Step 3 can be expressed as the following choice of $b^{(3)}(z)^{\prime}$ :

$$
b^{(3)}(z)^{\prime}=\left(\begin{array}{c}
\vartheta^{\prime} a^{\prime}\left(\beta_{0}^{\prime}-(z-1) \bar{\alpha}_{0}^{\prime} A_{1}\right) \\
\zeta^{\prime} a^{\prime} \beta_{0}^{\prime} \\
q^{\prime} \beta_{0}^{\prime} \\
\beta_{1}^{\prime}
\end{array}\right) \text {. }
$$

Remark 30 (Minimal basis). This step brings out from $\phi(z)^{\prime}$ some other cointegrating relations $\zeta^{\prime} a^{\prime} \beta_{0}^{\prime}$ that map the I(2) variables directly to I(0) without the help of first differences $\Delta$. Equation (36) shows how the canonical system of root functions can be reduced to minimal order.

Example 4 (Multicointegration coefficient in the span of $\beta_{2}$ ). Consider the special case when the multicointegrating coefficient $\bar{\alpha}_{0}^{\prime} A_{1}$ satisfies $\bar{\alpha}_{0}^{\prime} A_{1}=\bar{\alpha}_{0}^{\prime} A_{1} P_{\beta_{2}}$, i.e., it has components only in the direction of $\beta_{2}$. This special case is relevant, because $\beta_{2}^{\prime} \Delta x_{t} \sim I(1)$ while $\beta_{i}^{\prime} \Delta x_{t} \sim I(d)$ with $d \leq 0$ for $i=0,1$.

One can see that in this case the conditions in Proposition 6 are not satisfied. In fact (29) cannot hold, as $\bar{\alpha}_{0}^{\prime} A_{1} \bar{\beta}_{0}=0$. Step 2 is hence empty, and this implies that the rows including $q^{\prime}$ are missing and $a=I$ in $b^{(2)}(z)^{\prime}$ in (33) and (36).

Applying Step 3, Equation (34) is always satisfied by the choice $\rho^{\prime}=0^{\prime}, \tau^{\prime}=0^{\prime}$ because $\bar{\alpha}_{0}^{\prime} A_{1}\left(\bar{\beta}_{0}, \bar{\beta}_{1}\right)=0$. Equation (35) then reads $\zeta^{\prime} \bar{\alpha}_{0}^{\prime} A_{1} \bar{\beta}_{2}=0$, which is satisfied if and only if $\delta:=\bar{\alpha}_{0}^{\prime} A_{1} \bar{\beta}_{2}$ has reduced rank. In this case, let the rank factorization be $\delta=\psi \eta^{\prime}$, with $\psi$ and $\eta$ of full column rank. One can then let $\zeta^{\prime}=\psi_{\perp}^{\prime}$ and choose $\vartheta^{\prime}=\bar{\psi}^{\prime}$, so that

$$
b^{(3)}(z)^{\prime}=\left(\begin{array}{c}
\bar{\psi}^{\prime} \beta_{0}^{\prime}-(z-1) \eta^{\prime} \\
\zeta^{\prime} \beta_{0}^{\prime} \\
\beta_{1}^{\prime}
\end{array}\right) \text {. }
$$

There are several examples of this separation in the I(2) VAR literature; for example Kongsted (2005) discusses this when $r_{0}>r_{2}$.

\section{Conclusions}

This paper discusses the notion of cointegrating space for general $I(d)$ processes. The notion of cointegrating space was formally introduced in the literature by Johansen (1988) for the case of I(1) VAR system. The definition of the cointegrating space is simplest in the $I(1)$ case without multicointegration, because there is no need to consider vector polynomials in the lag operator. 
Engle and Yoo (1991) introduced the notion of polynomial cointegrating vectors in parallel with the related one of multicointegration in Granger and Lee (1989). However, the literature has not yet discussed the notion of cointegrating space in the general polynomial case; this paper fills this gap.

In this context, this paper recognises that cointegrating vectors are in general root functions, which have been analysed at length in the mathematical and engineering literature, see e.g., Gohberg et al. (1993). This allows to characterise a number of properties of cointegrating vectors.

Canonical systems of root functions are found to provide a basis of several notions of cointegration space in the multicointegrated case. The extended local rank factorization of Franchi and Paruolo (2016) can be used to explicitly derive a canonical system of root functions. This result is constructive, as it gives an explicit way to derive such a basis from the VAR polynomial.

The canonical system of root functions constructed in this way is not necessarily of minimal polynomial degree, however. The three-step procedure of Forney (1975) to reduce this basis to minimal-degree is reviewed and restated in terms of rank factorizations. The application of this procedure to I(2) VAR systems is shown to separate the polynomial and the non-polynomial cointegrating vectors.

Author Contributions: The authors contributed equally to the paper. All authors have read and agreed to the published version of the manuscript.

Funding: This research received no external funding.

Conflicts of Interest: The authors declare no conflict of interest.

\section{Appendix A}

\section{Appendix A.1. Scalar, Vector, Matrix Analytic Functions}

Consider a rational functions $a(z)$, defined as $a(z)=c(z) / d(z)$ with $c(z)$ and $d(z)$ polynomials, where $d(z) \not \equiv 0$. One can ask when $a(z)$ is analytic on $D\left(z_{\omega}, \eta\right), \eta>0$. The following remark states that this is the case provided $z_{\omega}$ is not a root of $d(z)$.

Remark A1 (Rational scalars can be analytic on $D\left(z_{\omega}, \eta\right)$ ). Let $a(z)$ be a rational function, i.e., $a(z)=c(z) / d(z)$ with $c(z)$ and $d(z)$ polynomial; assume also in addition that $d(z)$ has no root equal to $z_{\omega}$. Then $a(z)$ is analytic on $D\left(z_{\omega}, \eta\right)$, for some $\eta>0$. In fact, let $q=\operatorname{deg} d(z)$ be the degree of $d(z)$, and decompose $d(z)=\sum_{j=0}^{q} d_{j}^{\circ} z^{j}$ as $d(z)=d_{q}^{\circ} \prod_{j=1}^{n}\left(z-u_{j}\right)^{k_{j}}$, where $u_{j}$ are the roots of $d(z)$ with multiplicity $k_{j}, j=1, \ldots, n$ using the factor theorem for polynomials, see e.g., Barbeau (1989, p. 56). Then each term $\left(z-u_{j}\right)^{-k_{j}}$ has an analytic representation on $D\left(z_{\omega}, \eta\right)$, $\eta>0$, see e.g., Lemma A1 below. Note that this generates an infinite tail in $a(z)$, i.e., a $(z)$ is not polynomial in this case (unless $q=0$ ).

Lemma A1 (The inverse of a polynomial is analytic away form its roots). Let $u_{1}, \ldots, u_{n} \in \mathbb{C}$ be the distinct roots of a polynomial $d(z)$ with multiplicities $k_{1}, \ldots, k_{n}, k_{j} \in \mathbb{N}$, and let $v \in \mathbb{C}$ be another point, distinct from $u_{j}$; then one can pick some radius $\delta$ with $0<\delta<\min _{j=1, \ldots, n}\left|u_{j}-v\right|$ such that $d(z)^{-1}$ is analytic on $z \in D(v, \delta)$.

Proof. The polynomial $d(z)$ can be decomposed as $d(z)=a \prod_{j=1}^{n}\left(z-u_{j}\right)^{k_{j}}$. Next consider each term in the product $\left(z-u_{j}\right)^{k_{j}}$ and observe that $z-u_{j}=(z-v)-\left(u_{j}-v\right)=(v-$ $\left.u_{j}\right)\left(1-x_{j}\right)$ where $x_{j}:=(z-v) /\left(u_{j}-v\right)$. Define $0<\delta_{j}<\left|u_{j}-v\right|$ and note that $\left|x_{j}\right|<1$ for $z \in D\left(v, \delta_{j}\right)$, so that $\left(1-x_{j}\right)^{-1}=\sum_{s=0}^{\infty} x_{j}^{s}$ for $z \in D\left(v, \delta_{j}\right)$ and

$$
\left(z-u_{j}\right)^{-k_{j}}=\left(v-u_{j}\right)^{k_{j}}\left(\sum_{s=0}^{\infty}\left(\frac{z-v}{u_{j}-v}\right)^{s}\right)^{k_{j}} \quad z \in D\left(v, \delta_{j}\right) .
$$


Hence $\left(z-u_{j}\right)^{-k_{j}}$ is analytic on $z \in D\left(v, \delta_{j}\right)$ for any $j=1, \ldots, n$, and as a consequence also on $z \in D(v, \delta)$ with $0<\delta<\min _{j=1, \ldots, n}\left|u_{j}-v\right|$. This implies that $d(z)^{-1}=a^{-1} \prod_{j=1}^{n}(z-$ $\left.u_{j}\right)^{-k_{j}}$ is analytic on $z \in D(v, \delta)$.

Similarly, consider a $1 \times p$ vector function $\zeta(z)^{\prime}$ with rational entries. The denominator polynomials in all entries can be collected in a single one, the least common denominator, and hence $\zeta(z)^{\prime}$ has representation $\zeta(z)^{\prime}=\frac{1}{d(z)} b(z)^{\prime}$ where $d(z)$ is a monic polynomial and $b(z)^{\prime}$ is a $1 \times p$ vector polynomial, and $d(z)$ and $b(z)^{\prime}$ are relatively prime. The same applies to $p \times p$ rational matrix functions $C(z)$.

Example A1 (The least common denominator of bivariate rational vectors). The least common denominator can be illustrated as follows. Take a $1 \times 2$ rational row vector $a(z)^{\prime}=$ $\left(a_{1}(z), a_{2}(z)\right)=\left(c_{1}(z) / d_{1}(z), c_{2}(z) / d_{2}(z)\right)$, where $c_{i}(z), d_{i}(z)$ are (nonzero) polynomials $i=$ 1,2 ; then one can find a polynomial $d(z)$ with lowest degree such that $d(z)=h_{1}(z) d_{1}(z)=$ $h_{2}(z) d_{2}(z)$ where $h_{i}(z)$ are polynomials $i=1,2 ; d(z)$ is the least common multiple of the denominators, i.e., the least common denominator, and one has

$$
a(z)^{\prime}=\left(\frac{c_{1}(z)}{d_{1}(z)}, \frac{c_{2}(z)}{d_{2}(z)}\right)=\left(\frac{c_{1}(z) h_{1}(z)}{d(z)}, \frac{c_{2}(z) h_{2}(z)}{d(z)}\right)=: \frac{1}{d(z)} b(z)^{\prime}
$$

with $b(z)^{\prime}:=\left(b_{1}(z), b_{2}(z)\right):=\left(c_{1}(z) h_{1}(z), c_{2}(z) h_{2}(z)\right)$ where $b_{j}(z):=c_{j}(z) h_{j}(z)$ are still polynomials, so that $b(z)^{\prime}$ is a vector polynomial. The vector polynomial $b(z)^{\prime}$ and the scalar polynomial $d(z)$ are relatively prime, because there is no scalar polynomial $g(z)$ that divides both $d(z)$ and all the elements in $b(z)^{\prime}$. The polynomials in $b(z)^{\prime}$ and $d(z)$ can still be divided by a scalar in $F$, so $d(z)$ can be assumed to be monic.

Remark A2 (Rational vector and matrices). The $1 \times p$ analytic vector functions $\zeta(z)^{\prime}$ and $p \times p$ analytic matrix functions $C(z)$ can be generated as rational vectors or matrices, as long as their denominator polynomial $d(z)$ has no root equal to $z_{\omega}$. When $d(z)$ has one root equal to $z_{\omega}$ with multiplicity $m>0$, this implies that $\zeta(z)^{\prime}$ or $C(z)$ have a pole of order $m>0$ at $z_{\omega}$, and $\zeta(z)^{\prime}$ or $C(z)$ are not analytic on a disk $D\left(z_{\omega}, \eta\right)$ centered around $z_{\omega}$.

\section{Appendix A.2. Spans of Canonical Systems of Root Functions}

This section considers linear combinations of canonical system of root functions $\phi(z)^{\prime}$ with coefficients in $F, F[z]$ and $F(z)$. Attention is first given to multiplication of a root function by a rational or polynomial scalar; next generic linear combinations of canonical system of root functions in $\phi(z)^{\prime}$ are considered.

In order to discuss results, the notion of generalized root function is introduced first.

Definition A1 (Generalized root function). Let $n \in \mathbb{Z}$ and $\zeta(z)^{\prime}$ be a root function of $C(z)$ at $z_{\omega}$ and order s, see Definition 3; then

$$
\xi(z)^{\prime}:=\left(1-z / z_{\omega}\right)^{n} \zeta(z)^{\prime}
$$

is called a generalized root function of $C(z)$ at $z_{\omega}$ with order s and exponent $n$.

Observe that this is in line with Definition 5 of generalized cointegrating vectors for rational vectors. The reason for the introduction of the notion of generalized root function is provided by the next proposition.

Proposition A1 (Multiplication by a scalar). Let $\zeta(z)^{\prime}$ be a $1 \times p$ root function for $C(z)$ of order s on $D\left(z_{\omega}, \eta\right), \eta>0$. Then

(i) if $a \in F, a \neq 0$, then $a \zeta(z)^{\prime}$ is a root function on $D\left(z_{\omega}, \eta\right)$ of order s;

(ii) if $a(z) \in F[z], a(z) \neq 0$, then $a(z) \zeta(z)^{\prime}$ is a generalized root function on $D\left(z_{\omega}, \eta\right)$ of order $s$ and exponent $n \in \mathbb{N}_{0}:=\mathbb{N} \cup 0$; 
(iii) if $a(z) \in F(z), a(z) \neq 0$, then $a(z) \zeta(z)^{\prime}$ is a generalized root function on $D\left(z_{\omega}, \eta\right)$ of order $s$ and exponent $n \in \mathbb{Z}$.

Proof. Consider first case $($ iii $)$.

(iii). $a(z)=a_{1}(z) / a_{2}(z)$ where $a_{i}(z)$ are relatively prime polynomials, $i=1,2$. If $a_{i}(z)$ has root $z_{\omega}$ then it admits representation $a_{i}(z)=\left(1-z / z_{\omega}\right)^{n_{i}} a_{i}^{\diamond}(z)$ with $n_{i} \in \mathbb{N}_{0}$ and $a_{i}^{\diamond}\left(z_{\omega}\right) \neq$ $0, i=1$, 2. Hence $a(z)=a_{1}(z) / a_{2}(z)=\left(1-z / z_{\omega}\right)^{n_{1}-n_{2}} a^{\diamond}(z)$ where $a^{\diamond}(z):=a_{1}^{\diamond}(z) / a_{2}^{\diamond}(z)$ with $a^{\diamond}\left(z_{\omega}\right) \neq 0$ and $a(z) \zeta(z)^{\prime}=\left(1-z / z_{\omega}\right)^{n_{1}-n_{2}} a^{\diamond}(z) \zeta(z)^{\prime}$. The factor $\left(1-z / z_{\omega}\right)^{n_{1}-n_{2}}$ has exponent $n_{1}-n_{2}$, which can be positive, negative or 0 ; because $a_{i}(z)$ are relatively prime polynomials, $i=1,2$, either $n_{1}>0$ or $n_{2}>0$ or $n_{1}=n_{2}=0$. The factor $a^{\diamond}(z) \zeta(z)^{\prime}$ is a generalized root function of order $s$, because $\zeta(z)^{\prime}$ is a root function of order $s$ and the scalar factor $a^{\diamond}(z)$ satisfies $a^{\diamond}\left(z_{\omega}\right) \neq 0$, so that $a^{\diamond}\left(z_{\omega}\right) \zeta\left(z_{\omega}\right)^{\prime} \neq 0^{\prime}$. This shows that $a(z) \zeta(z)^{\prime}$ is a generalized root function of order $s$ and exponent $n=n_{1}-n_{2}$.

(ii). Set $a_{2}(z)=1$ in the proof of $\left.i i i\right)$, and note that the exponent is $n_{1}$, which is either 0 or positive.

(i). Set $a_{1}(z)=a, a_{2}(z)=1$ in the proof of $\left.i i i\right)$, and note that the exponent is $n_{1}=0$.

Remark A3 (A generalized root function is meromorphic). A generalized root function $\xi(z)^{\prime}$ is analytic on $D\left(z_{\omega}, \eta\right)$ except for the possibility to have poles at the isolated point $z_{\omega}$, i.e., it is a meromorphic function on $D\left(z_{\omega}, \eta\right)$.

Remark A4 (A generalized root function can be analytic). When the exponent $n$ of $\xi(z)^{\prime}$ is zero, the generalized root function $\xi(z)^{\prime}$ coincides with the root function $\zeta(z)^{\prime}$. When the exponent $n$ of $\xi(z)^{\prime}$ is positive, then the generalized root function $\xi(z)^{\prime}$ has a zero at $z_{\omega}$. In both cases $\xi(z)^{\prime}$ is analytic. So a generalized root function can be analytic (with or without a zero at $z_{\omega}$ ).

Remark A5 (Generalized root function and cointegration). Observe that Definition A1 implies the following: given a meromorphic function $\xi(z)^{\prime}$, check if it has a root or a pole at $z_{\omega}$; this function is a generalized root functions if, after removing the pole or the zero at $z_{\omega}$ by multiplying it by $\left(1-z / z_{\omega}\right)^{-n}$ where $n$ is the order of the root or of the pole, the resulting function is a root function, i.e., a cointegrating vector. This is in line with Definition 5.

Attention is now turned to linear combinations of a canonical system of root functions $\phi(z)^{\prime}$. The scalars of the linear combination can be in $F, F[z]$ or $F(z)$. The main result in Proposition A2 below is that $F[z]$-linear combinations of $\phi(z)^{\prime}$ generate a generalized root function possibly with a zero at $z_{\omega}$, while $F(z)$-linear combinations of $\phi(z)^{\prime}$ generate a generalized root function possibly with a pole or a zero at $z_{\omega}$.

In the following, let $v^{\prime}=\left(v_{1}, \ldots, v_{m}\right)^{\prime} \in F^{m}$ be a $1 \times m$ vector with elements in $F$. Let also $A_{v}$ be the set of non-zero entries in $v, A_{v}:=\left\{i: v_{i} \neq 0\right\}$, with $n_{v}$ the cardinality of $A_{v}$ and $\left(i_{1}, \ldots, i_{n_{v}}\right)$ the ordered set of indices in $A_{v}, i_{1}<\cdots<i_{n_{v}}, i_{j} \in A_{v}$. Similarly, let $w(z)^{\prime}=\left(w_{1}(z), \ldots, w_{m}(z)\right)^{\prime} \in F[z]^{m}$ be a $1 \times m$ vector with polynomial elements in $w_{i}(z) \in F[z]$ with $\left(j_{1}, \ldots, j_{n_{w}}\right)$ its ordered set of indices of nonzero elements in $A_{w}:=\left\{i: w_{i}(z) \neq 0\right\}$, and let finally $u(z)^{\prime}=\left(u_{1}(z), \ldots, u_{m}(z)\right)^{\prime} \in F(z)^{m}$ be a $1 \times m$ vector with rational elements in $u_{i}(z) \in F(z)$ with $\left(k_{1}, \ldots, k_{n_{u}}\right)$ as its ordered set of indices of nonzero elements in $A_{u}:=\left\{i: u_{i}(z) \neq 0\right\}$.

Proposition A2 (Linear combinations). Let $\phi(z)^{\prime}=\left(\phi_{1}(z), \ldots \phi_{m}(z)\right)^{\prime}$ be a canonical system of root functions of $C(z)$ on a disc $D\left(z_{\omega}, \eta\right), \eta>0$ with orders $s_{1}, \ldots, s_{m}$; let also $v^{\prime} \in F^{m}$, $w(z)^{\prime} \in F[z]^{m}$ and $u(z)^{\prime} \in F(x)^{m}$ be nonzero vectors; one has:

(i) $v^{\prime} \phi(z)^{\prime}=\sum_{i=1}^{m} v_{i} \phi_{i}(z)^{\prime}$ is a root function of order $s=\min _{i \in A_{v}} s_{i}$;

(ii) $w(z)^{\prime} \phi(z)^{\prime}=\sum_{j=1}^{m} w_{j}(z) \phi_{j}(z)^{\prime}$ is a generalized root function, with exponent $q=\min _{j \in A_{w}}$ $\left(q_{j}\right) \geq 0$ where $q_{j}$ is the order of $z_{\omega}$ as a zero of $w_{j}(z)$, and with order $s:=\min _{j \in A_{w}}\left(q_{j}-q+\right.$ $\left.s_{j}\right)>0$; 
(iii) $u(z)^{\prime} \phi(z)^{\prime}=\sum_{k=1}^{m} u_{k}(z) \phi_{k}(z)^{\prime}$ is a generalized root function, possibly with a pole or a zero at $z_{\omega}$, with exponent $q=\min _{k \in A_{u}}\left(q_{k}\right) \in \mathbb{Z}$ where $q_{k}$ is the order of $z_{\omega}$ as a pole or as a zero of $u_{k}(z)$, and with order $s=\min _{k \in A_{u}}\left(q_{k}-q+s_{k}\right)>0$.

Proof. (i). By definition $\phi_{i}(z)^{\prime}=\sum_{j=0}^{s_{i}}\left(z-z_{\omega}\right)^{j} \phi_{i j}^{\prime}$, analytic on $D\left(z_{\omega}, \eta\right)$ and $\phi_{i j}^{\prime} \in F^{p}$. One finds $\sum_{i=1}^{m} v_{i} \phi_{i}(z)^{\prime}=\sum_{j=0}^{s_{i}}\left(z-z_{\omega}\right)^{j} \varphi_{j}^{\prime}$ with $\varphi_{j}^{\prime}:=\sum_{i=1}^{m} v_{i} \phi_{i j}^{\prime} \in F^{p}$ because $F$ is a field, and hence it is closed under multiplication. Hence $v^{\prime} \phi(z)^{\prime}$ is a polynomial with coefficients vectors in $F^{p}$, of the same form as each $\phi_{i}(z)^{\prime}$, and one finds that

$$
v^{\prime} \phi(z)^{\prime} C(z)=\sum_{i=1}^{m} v_{i} \phi_{i}(z)^{\prime} C(z)=\sum_{i \in A_{v}} v_{i}\left(z-z_{\omega}\right)^{s_{i}} \widetilde{\phi}_{i}(z)^{\prime}=\left(z-z_{\omega}\right)^{s} \widetilde{\phi}(z)^{\prime}
$$

where $s:=\min \left\{s_{i_{1}}, \ldots, s_{i_{v}}\right\}, \widetilde{\phi}(z)^{\prime}:=\sum_{h=1}^{n_{v}}\left(z-z_{\omega}\right)^{s_{h}-s_{v_{i}}} \widetilde{\phi}_{i_{h}}(z)^{\prime}$ and $\widetilde{\phi}_{i_{h}}\left(z_{\omega}\right)^{\prime} \neq 0^{\prime}$. Note that because $v^{\prime}$ is a nonzero vector, the set $A_{v}$ is not empty. Next observe that $\widetilde{\phi}\left(z_{\omega}\right)^{\prime} \neq 0^{\prime}$ otherwise this would contradict the property of $\phi_{i_{h}}(z)^{\prime}$ to be of maximal order and linearly independent from the previous root function $\phi_{i}(z)^{\prime}$ for $i<i_{h}$. This shows that $v^{\prime} \phi(z)^{\prime}$ is a root function of order $s$.

(ii). Consider $w(z)^{\prime} \phi(z)^{\prime}=\sum_{i=1}^{m} w_{i}(z) \phi_{i}(z)^{\prime}$, where by Proposition A1. (i), one has that $w_{i}(z) \phi_{i}(z)^{\prime}$ is a generalized root function with representation $w_{i}(z) \phi_{i}(z)^{\prime}=(1-$ $\left.z / z_{\omega}\right)^{q_{i}} w_{i}^{\diamond}(z) \phi_{i}(z)^{\prime}$ say, with $q_{i} \geq 0$ and $w_{i}^{\diamond}(z) \phi_{i}(z)^{\prime}$ a root function of order $s_{i}$. Let $q:=$ $\min \left(q_{j_{1}}, \ldots, q_{j_{w}}\right)$, and note that $w(z)^{\prime} \phi(z)^{\prime}=\left(1-z / z_{\omega}\right)^{q} \zeta(z)^{\prime}$ with $\zeta(z)^{\prime}:=\sum_{h=1}^{n_{w}}(1-$ $\left.z / z_{\omega}\right)^{q_{j_{h}}-q} w_{j_{h}}^{\diamond}(z) \phi_{j_{h}}(z)^{\prime}$. In order to show that $\zeta\left(z_{\omega}\right)^{\prime} \neq 0^{\prime}$, let $B_{w}$ be the set of indices $j \in A_{w}$ with $q_{j}=q$, and observe that $\zeta\left(z_{\omega}\right)^{\prime}=\sum_{j \in B_{w}} w_{j}^{\diamond}\left(z_{\omega}\right) \phi_{j}\left(z_{\omega}\right)^{\prime}$ where $w_{j}^{\diamond}\left(z_{\omega}\right) \neq 0$ by construction and $\phi_{j}\left(z_{\omega}\right)^{\prime} \neq 0^{\prime}$ by the definition of root function. If $\zeta\left(z_{\omega}\right)^{\prime}=0^{\prime}$ this would imply that there is a nonzero linear combination of $\phi\left(z_{\omega}\right)^{\prime}$ equal to $0^{\prime}$, i.e., that $\phi\left(z_{\omega}\right)^{\prime}$ is not of full row rank, which contradicts the construction in Definition 4 . This implies that $\zeta\left(z_{\omega}\right)^{\prime} \neq 0^{\prime}$, and that $w(z)^{\prime} \phi(z)^{\prime}$ is a generalized root function of order $q$.

Next, because $\phi_{j}(z)^{\prime}$ is a root function of order $s_{j}$ one has

$$
\begin{aligned}
\zeta(z)^{\prime} C(z) & =\sum_{h=1}^{n_{w}}\left(1-z / z_{\omega}\right)^{q_{j_{h}}-q} w_{j_{h}}^{\diamond}(z) \phi_{j_{h}}(z)^{\prime} C(z) \\
& =\sum_{h=1}^{n_{w}}\left(1-z / z_{\omega}\right)^{q_{j_{h}}-q+s_{j_{h}}} w_{j_{h}}^{\diamond}(z) \widetilde{\phi}_{j_{h}}(z)^{\prime}=\left(1-z / z_{\omega}\right)^{s} \widetilde{\zeta}(z)^{\prime}
\end{aligned}
$$

where $\widetilde{\zeta}(z)^{\prime}:=\sum_{h=1}^{n_{w}}\left(1-z / z_{\omega}\right)^{q_{j_{h}}-q+s_{j_{h}}-s} w_{j_{h}}^{\diamond}(z) \widetilde{\phi}_{j_{h}}(z)^{\prime}$. Finally, in order to prove that the order of the generalized root function is s, one needs to show that $\widetilde{\zeta}\left(z_{\omega}\right)^{\prime} \neq 0^{\prime}$. Let $C_{w}$ be the set of indices $j \in A_{w}$ with $q_{j_{h}}-q+s_{j_{h}}=s$, and observe that $\widetilde{\zeta}\left(z_{\omega}\right)^{\prime}:=\sum_{j \in C_{w}} w_{j}^{\diamond}\left(z_{\omega}\right) \widetilde{\phi}_{j}\left(z_{\omega}\right)^{\prime}$ where $w_{j}^{\diamond}\left(z_{\omega}\right) \neq 0$ and $\phi_{j}\left(z_{\omega}\right)^{\prime} \neq 0^{\prime}$ as above. If $\widetilde{\zeta}\left(z_{\omega}\right)^{\prime}=0^{\prime}$, then there exists a nonzero linear combination of $\phi\left(z_{\omega}\right)^{\prime}$ equal to $0^{\prime}$, which would imply the existence of a root function of higher order obtained by combination of the rows in $\phi(z)^{\prime}$ with index $C_{w}$, which contradict the fact that the orders are chosen to be maximal. This implies that the order of the generalized root function is equal to $s$.

(iii). The proof is the same as in $i i)$. Note that here $q_{i}$ may be negative.

Remark A6 (Closure with respect to linear combinations). Proposition A2 shows that $F[z]$ linear combinations and $F(z)$-linear combinations of a canonical systems of root functions $\phi(z)^{\prime}$ produce generalized root functions. Note that $\phi(z)^{\prime}$ is itself a set of generalized root functions (with 0 exponent). Hence, in this sense, generalized root functions are closed under $F[z]$-linear combinations and $F(z)$-linear combinations. 
Remark A7 (Spans). Indicate the set of G-linear combinations $\phi(z)^{\prime}$ as $\operatorname{row}_{G}\left(\phi(z)^{\prime}\right)$, where $G=F, F(z), F[z]$. It is simple to observe that

$$
\operatorname{row}_{F}\left(\phi(z)^{\prime}\right) \subset \operatorname{row}_{F[z]}\left(\phi(z)^{\prime}\right) \subset \operatorname{row}_{F(z)}\left(\phi(z)^{\prime}\right) .
$$

Remark A8 (Role of characteristics of canonical system of root functions). The proof of Proposition A2 reveals that, in order to conclude that a $F[z]-$ or $F(z)$-linear combination of $\phi(z)^{\prime}$ is a generalized root function, the property that $\phi\left(z_{\omega}\right)^{\prime}$ is of full row rank plays a crucial role. In fact, when reaching the equality $w(z)^{\prime} \phi(z)^{\prime}=\left(1-z / z_{\omega}\right)^{9} \zeta(z)^{\prime}$ where $q$ is the exponent of the linear combination, one can show that $\zeta\left(z_{\omega}\right)^{\prime} \neq 0^{\prime}$ by making use of this property only, without using the maximal orders of the root functions in $\phi(z)^{\prime}$. This proves the following corollary.

Corollary A1 (Linear combinations of a set of root functions). Replace the canonical system of root functions $\phi(z)^{\prime}$ in Proposition $A 2$ with a set of $m$ root functions $\xi(z)^{\prime}$ for $C(z)$ on $D\left(z_{\omega}, \eta\right)$, $\eta>0$ such that $\xi\left(z_{\omega}\right)^{\prime}$ is of full row rank; then the $F[z]$ - or $F(z)$-linear combinations $w(z)^{\prime} \xi(z)^{\prime}$ and $u(z)^{\prime} \xi(z)^{\prime}$ are generalized root functions with the same exponents as in Proposition A2.

\section{Appendix A.3. Truncations of Root Functions}

This section discusses how the truncation of a root function still delivers a root function, possibly of lower order. The main implication of this property is that one can take any element in $\operatorname{row}_{G}\left(\phi(z)^{\prime}\right)$ for $G=F, F[z], F(z)$ and obtain other root functions by truncation, thus enlarging the set of root functions that can be generated from $\operatorname{row}_{G}\left(\phi(z)^{\prime}\right)$.

Let $\zeta(z)^{\prime}:=\sum_{j=0}^{\infty}\left(z-z_{\omega}\right)^{j} \zeta_{j}^{\prime}$ be a $1 \times p$ root function of order $s$ of $C(z)$ on $D\left(z_{\omega}, \eta\right)$, $\eta>0$, and indicate the truncation of $\zeta(z)^{\prime}$ to a polynomial of degree $r$ as $\zeta^{(r)}(z)^{\prime}:=$ $\sum_{j=0}^{r}\left(z-z_{\omega}\right)^{j} \zeta_{j}^{\prime}$; the remainder $\zeta(z)^{\prime}-\zeta^{(r)}(z)^{\prime}=\sum_{j=r+1}^{\infty}\left(z-z_{\omega}\right)^{j} \zeta_{j}^{\prime}$ is called the tail of $\zeta(z)^{\prime}$. The following proposition clarifies that one can modify the tail of a root function without affecting its property to factor some power of $\left(1-z / z_{\omega}\right)$ from $C(z)$. One special case is that one can delete the tail after the order $s$ of the root function without changing its order.

Proposition A3 (Truncations). Let $\zeta(z)^{\prime}:=\sum_{j=0}^{\infty}\left(z-z_{\omega}\right)^{j} \zeta_{j}^{\prime}$ be a root function of order s for $C(z)=\sum_{j=0}^{\infty}\left(z-z_{\omega}\right)^{j} C_{j}$ on $D\left(z_{\omega}, \eta\right), \eta>0, \zeta\left(z_{\omega}\right)^{\prime} \neq 0^{\prime}$, and let $\psi(z)^{\prime}:=\sum_{j=0}^{\infty}\left(z-z_{\omega}\right)^{j} \psi_{j}^{\prime}$ be $a 1 \times$ vector function, analytic on $D\left(z_{\omega}, \eta\right)$; then

(i) for an integer $\ell \geq 1$, the $1 \times p$ row vector $\xi(z)^{\prime}$ with

$$
\xi(z)^{\prime}:=\zeta(z)^{\prime}+\left(z-z_{\omega}\right)^{\ell} \psi(z)^{\prime}
$$

is still a root function on $D\left(z_{\omega}, \eta\right)$ of order $n \geq \min (\ell, s)$;

(ii) if one chooses $\ell \leq s$, in the definition (A3) of $\xi(z)^{\prime}$ with $\psi(z)^{\prime}$ proportional to the tail of $\zeta(z)^{\prime}$, a special case of $i)$ is that the truncation of $\zeta(z)^{\prime}$ to the polynomial $\zeta^{(\ell)}(z)^{\prime}:=\sum_{j=0}^{\ell}\left(z-z_{\omega}\right)^{j} \zeta_{j}^{\prime}$ of order $\ell$ is also a root function on $D\left(z_{\omega}, \eta\right)$ of order $n \geq \ell$;

(iii) finally if $\zeta(z)^{\prime}:=\sum_{j=0}^{\infty}\left(z-z_{\omega}\right)^{j} \zeta_{j}^{\prime}$ is a root function of order s in a canonical system of root functions of $C(z)$ at $z_{\omega}$, then its truncation $\zeta^{(s-1)}(z)^{\prime}:=\sum_{j=0}^{s-1}\left(z-z_{\omega}\right)^{j} \zeta_{j}^{\prime}$ to a polynomial of degree $s-1$ is still a root function of $C(z)$ at $z_{\omega}$ on $D\left(z_{\omega}, \eta\right)$ of order $s$.

Proof. (i). By definition one has $\zeta(z)^{\prime} C(z)=\left(z-z_{\omega}\right)^{s} \widetilde{\zeta}(z)^{\prime}$ with $\widetilde{\zeta}\left(z_{\omega}\right)^{\prime}=\sum_{h=0}^{s} \zeta_{h}^{\prime} C_{s-h} \neq$ $0^{\prime}$. Hence, setting $q:=\min (\ell, s)$, one finds

$$
\xi(z)^{\prime} C(z)=\zeta(z)^{\prime} C(z)+\left(z-z_{\omega}\right)^{\ell} \psi(z)^{\prime} C(z)=\left(z-z_{\omega}\right)^{q} \widetilde{\zeta}(z)^{\prime} .
$$

where $\widetilde{\zeta}(z)^{\prime}=\left(z-z_{\omega}\right)^{s-q} \widetilde{\zeta}(z)^{\prime}+\left(z-z_{\omega}\right)^{\ell-q} \psi(z)^{\prime} C(z)$. If $\widetilde{\xi}\left(z_{\omega}\right)^{\prime} \neq 0^{\prime}$, then $\xi(z)^{\prime}$ is a root function of order $q$. If, instead, $\widetilde{\xi}\left(z_{\omega}\right)^{\prime}=0^{\prime}$, then $\widetilde{\xi}(z)^{\prime}$ is a root function of order $n$ greater than $q$; in any case $n \geq q$, with $n$ finite by Proposition 2 . 
(ii). Choose $\psi(z)^{\prime}=\left(z-z_{\omega}\right)^{-\ell}\left(\zeta(z)^{\prime}-\zeta^{(\ell)}(z)^{\prime}\right)=\sum_{j=\ell+1}^{\infty}\left(z-z_{\omega}\right)^{j-\ell} \zeta_{j}^{\prime}$ in (A3), so that $\xi(z)^{\prime}=\zeta^{(\ell)}(z)^{\prime}$. The statement follows from $(i)$.

(iii). The coefficients $\zeta(z)^{\prime}$ and $\zeta^{(s-1)}(z)^{\prime}$ generate the same coefficients $W_{h}:=\sum_{j=0}^{h} \zeta_{j}^{\prime} C_{h-j}$ for $h=0, \ldots, s-1$ in the convolution $\zeta(z)^{\prime} C(z)=W(z):=\sum_{h=0}^{\infty}\left(z-z_{\omega}\right)^{h} W_{h}$, where $W_{h}=0$ for $h=0, \ldots, s-1$ by definition of order $s$, see (8). This implies that $\zeta^{(s-1)}(z)^{\prime}$ is a root function at least of order $s$. However, because root functions in a canonical system of root functions are chosen of maximal order, the order of $\zeta^{(s-1)}(z)^{\prime}$ is equal to $s$. This completes the proof.

Remark A9 (Truncated cointegrating vectors). Proposition A3. (ii) implies that truncating a cointegrating vector to order $\ell<s$ preserves the cointegrating property, but not necessarily the order s.

Remark A10 (Cointegrating vectors in $I_{\omega}(1)$ VAR can be chosen not to be polynomial). Consider Example 1, where the orders of integration of (polynomial) linear combinations can be either 1 or 0 . In this case, root function are of order at most $s=1$, and Proposition A3. (iii) ensures that the root functions can be truncated to order $s-1=0$, i.e., to non-polynomial linear combinations.

Remark A11 (A generic $I_{\omega}(1)$ process may have polynomial cointegration relations). Consider now the generic case of an I(1) process. The orders of integration of (polynomial) linear combinations can be $0,-1,-2, \cdots-d$ say, with $d>0$. In this case, root function are of order at most $s=1,2 \ldots, d+1$, and Proposition A3. (iii) ensures that the root functions can be truncated to order $d$. If $d>0$ this may require polynomial linear combinations also in the $I_{\omega}(1)$ case.

Remark A12 (Polynomial cointegration vectors in $I_{\omega}(2)$ VAR can be chosen of order at most 1). Consider Example 2, where the orders of integration of (polynomial) linear combinations can be either 2, 1 or 0 . In this case, root function are of order at most $s=2$, and Proposition A3. (iii) ensures that the root functions can be truncated to order $s-1=1$, i.e., to polynomial linear combinations of order 1.

Remark A13 (Multicointegrated systems require polynomial cointegration relations). As shown in the previous three remarks, in general, multicointegrated systems require to consider polynomial linear combinations.

\section{Notes}

$1 \quad$ See Engle and Granger (1987, pp. 253-54). Here $N$ in their notation is replaced by $p$ and $\alpha$ with $\beta$ for consistency with the rest of the paper.

2 The following notation is employed: $F=\mathbb{R}, \mathbb{C}$ indicates either the field of real numbers $\mathbb{R}$ or the field of complex numbers $\mathbb{C}$ and if a matrix $A=\left(a_{1}, \ldots, a_{n}\right)$ is written in terms of its columns, $\operatorname{col}_{F}(A)$ indicates the column span of $A$ with coefficients in $F$, i.e., $\operatorname{col}_{F}(A):=\left\{v: v=\sum_{j=1}^{n} c_{i} a_{i}, c_{i} \in F\right\}$ and $\operatorname{row}_{F}\left(A^{\prime}\right)$ denotes the row span of $A^{\prime}$ with coefficients in $F$, i.e., $\operatorname{row}_{F}\left(A^{\prime}\right):=\left\{v^{\prime}\right.$ : $\left.v^{\prime}=\sum_{j=1}^{n} c_{i} a_{i}^{\prime}, c_{i} \in F\right\}$, where $A^{\prime}$ indicates the conjugate transpose of $A$. Hence $v \in \operatorname{col}_{F}(A)$ if and only if $v^{\prime} \in \operatorname{row}_{F}\left(A^{\prime}\right)$, i.e., the spaces coincide but the former contains column vectors while the latter contains row vectors. Here the row form is employed. $\varepsilon_{t}$ could be taken to be non-autocorrelated instead of i.i.d. with no major changes in the results in the paper.

4 This result is usually stated as $x_{t}=u_{t}-a_{0}$ where $a_{0}:=x_{0}-u_{0}$ is a generic constant, see e.g., Hannan and Deistler (1988) Equation (1.2.15).

5 In fact, substituting $C(z)=C+\widetilde{C}(z)(1-z)$, one finds $\zeta^{\prime} \Delta x_{t}=\zeta^{\prime} C \varepsilon_{t}+\zeta^{\prime} \widetilde{C}(L) \Delta \varepsilon_{t}$, and applying $\mathcal{S}$ to both sides gives $\zeta^{\prime} x_{t}-$ $\zeta^{\prime} x_{0}=\zeta^{\prime} C \mathcal{S} \varepsilon_{t}+u_{t}-u_{0}$ where $u_{t}:=\zeta^{\prime} \widetilde{C}(L) \varepsilon_{t}$ is stationary. The term $\mathcal{S} \varepsilon_{t}$ is a bilateral random walk (Franchi and Paruolo 2019), a nonstationary process, so that the l.h.s. can be made stationary if and only if the coefficient $\zeta^{\prime} C$ loading $\mathcal{S} \varepsilon_{t}$ is 0 .

6 Theorem 3 provides two constructions of the local Smith factorization.

7 In this case $A(z)$ is analytic for all $z \in \mathbb{C}$.

8 In the first sentence in Definition 3.1 of Franchi and Paruolo (2019) ' $r_{0}^{\max }:=p-r_{0}$ ' should read ' $r_{0}^{\max }:=p^{\prime}$. The results of Franchi and Paruolo (2019, Theorem 3.3) are applied setting $F(z)$ there equal to $A(z)$ here. 
9 The present statement follows by Franchi and Paruolo (2019, Theorem 3.5) with $F(z)$ and $\Phi(z)$ there equal to $A(z)$ and $\Xi(z)^{-1}$ here.

\section{References}

Barbeau, Edward J. 1989. Polynomials. Berlin and Heidelberg: Springer.

Bauer, Dietmar, and Martin Wagner. 2012. A State Space Canonical Form for Unit Root Processes. Econometric Theory 28: 1313-49.

Beare, Brendan, and Won-ki Seo. 2020. Representation of I(1) and I(2) autoregressive Hilbertian processes. Econometric Theory 36: 773-802.

Beare, Brendan, Juwon Seo, and Won-ki Seo. 2017. Cointegrated Linear Processes in Hilbert Space. Journal of Time Series Analysis 38: 1010-27.

Engle, Robert F., and Clive W. J. Granger. 1987. Co-integration and Error Correction: Representation, Estimation, and Testing. Econometrica 55: 251-76.

Engle, Robert F., and Sam B. Yoo. 1991. Cointegrated economic time series: An overview with new results. In Long-Run Economic Relations: Readings in Cointegration. Edited by Robert Engle and Clive Granger. Oxford: Oxford University Press, pp. 237-66.

Engsted, Tom, and Søren Johansen. 2000. Granger's Representation Theorem and Multicointegration. In Cointegration, Causality and Forecasting: Festschrift in Honour of Clive Granger. Edited by R. F. Engle and H. White. Oxford: Oxford University Press, pp. 200-12.

Forney, G. David, Jr. 1975. Minimal bases of rational vector spaces, with applications to multivariable linear systems. SIAM Journal on Control 13: 493-520. [CrossRef]

Franchi, Massimo, and Paolo Paruolo. 2011. Inversion of regular analytic matrix functions: Local Smith form and subspace duality. Linear Algebra and Its Applications 435: 2896-912.

Franchi, Massimo, and Paolo Paruolo. 2016. Inverting a matrix function around a singularity via local rank factorization. SIAM Journal of Matrix Analysis and Applications 37: 774-97.

Franchi, Massimo, and Paolo Paruolo. 2019. A general inversion theorem for cointegration. Econometric Reviews 38: 1176-201. [CrossRef]

Franchi, Massimo, and Paolo Paruolo. 2020. Cointegration in functional autoregressive processes. Econometric Theory 36: 803-39.

Gohberg, Israel, Marinus A. Kaashoek, and Frederik Van Schagen. 1993. On the local theory of regular analytic matrix functions. Linear Algebra and Its Applications 182: 9-25.

Granger, Clive W. J., and Tae-Hwy Lee. 1989. Investigation of production, sales and inventory relationships using multicointegration and non-symmetric error correction models. Journal of Applied Econometrics 4: S145-59.

Gregoir, Stèphane M. 1999. Multivariate time series with various hidden unit roots, Part I. Econometric Theory 15: 435-68.

Hannan, Edward J., and Manfred Deistler. 1988. The Statistical Theory of Linear Systems. Hoboken: John Wiley \& Sons.

Howlett, Phil G. 1982. Input retrieval in finite dimensional linear systems. Journal of Australian Mathematical Society (Series B) 23 : 357-82.

Hungerford, Thomas W. 1980. Algebra. Berlin and Heidelberg: Springer.

Hylleberg, Svend, Robert F. Engle, Clive W. J. Granger, and Sam B. Yoo. 1990. Seasonal integration and cointegration. Journal of Econometrics 44: 215-38.

Johansen, Søren. 1988. Statistical Analysis of Cointegration Vectors. Journal of Economic Dynamics and Control 12: 231-54.

Johansen, Søren. 1991. Estimation and Hypothesis Testing of Cointegration Vectors in Gaussian Vector Autoregressive Models. Econometrica 59: 1551-80.

Johansen, Søren. 1992. A representation of vector autoregressive processes integrated of order 2. Econometric Theory 8: 188-202.

Johansen, Søren. 1995. Identifying restrictions of linear equations with applications to simultaneous equations and cointegration. Journal of Econometrics 69: 111-32.

Johansen, Søren. 1996. Likelihood-Based Inference in Cointegrated Vector Auto-Regressive Models. Oxford: Oxford University Press.

Johansen, Søren, and E. Schaumburg. 1998. Likelihood analysis of seasonal cointegration. Journal of Econometrics 88: 301-39.

Kongsted, Hans Christian. 2005. Testing the nominal-to-real transformation. Journal of Econometrics 124: 205-25. [CrossRef]

Mosconi, Rocco, and Paolo Paruolo. 2017. Identification conditions in simultaneous systems of cointegrating equations with integrated variables of higher order. Journal of Econometrics 198: 271-76. [CrossRef]

Seo, Won-ki. 2019. Cointegration and Representation of Cointegrated Autoregressive Processes in Banach Spaces. arXiv arXiv:1712.08748v4. 